\title{
Drag reduction mechanisms on a generic square-back vehicle using an optimised yaw-insensitive base cavity
}

\author{
Magnus Urquhart ${ }^{1}\left[\right.$ Dax Varney $^{2} \cdot$ Simone Sebben $^{1} \cdot$ Martin Passmore $^{2}$
}

Received: 4 June 2021 / Revised: 3 October 2021 / Accepted: 6 October 2021 / Published online: 11 November 2021

(C) The Author(s) 2021

\begin{abstract}
Regulations on global greenhouse gas emission are driving the development of more energy-efficient passenger vehicles. One of the key factors influencing energy consumption is the aerodynamic drag where a large portion of the drag is associated with the base wake. Environmental conditions such as wind can increase the drag associated with the separated base flow. This paper investigates an optimised yaw-insensitive base cavity on a square-back vehicle in steady crosswind. The test object is a simplified model scale bluff body, the Windsor geometry, with wheels. The model is tested experimentally with a straight cavity and a tapered cavity. The taper angles have been optimised numerically to improve the robustness to side wind in relation to drag. Base pressures and tomographic Particle Image Velocimetry of the full wake were measured in the wind tunnel. The results indicate that a cavity decreases the crossflow within the wake, increasing base pressure, therefore lowering drag. The additional optimised cavity tapering further reduces crossflow and results in a smaller wake with less losses. The overall wake unsteadiness is reduced by the cavity by minimising mixing in the shear layers as well as dampening wake motion. However, the coherent wake motions, indicative of a balanced wake, are increased by the investigated cavities.
\end{abstract}

Magnus Urquhart

magnus.urquhart@chalmers.se

Max Varney

m.varney@lboro.ac.uk

Simone Sebben

simone.sebben@chalmers.se

Martin Passmore

m.a.passmore@lboro.ac.uk

1 Chalmers University of Technology, Gothenburg, Sweden

2 Loughborough University, Loughborough, England, UK 


\section{Graphical abstract}

\section{Square-back}

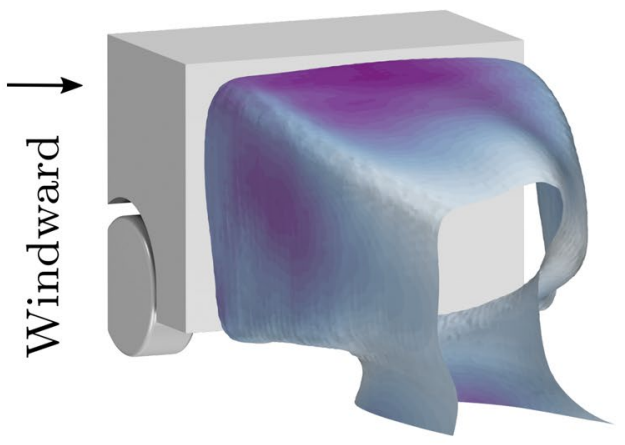

\section{Tapered cavity}

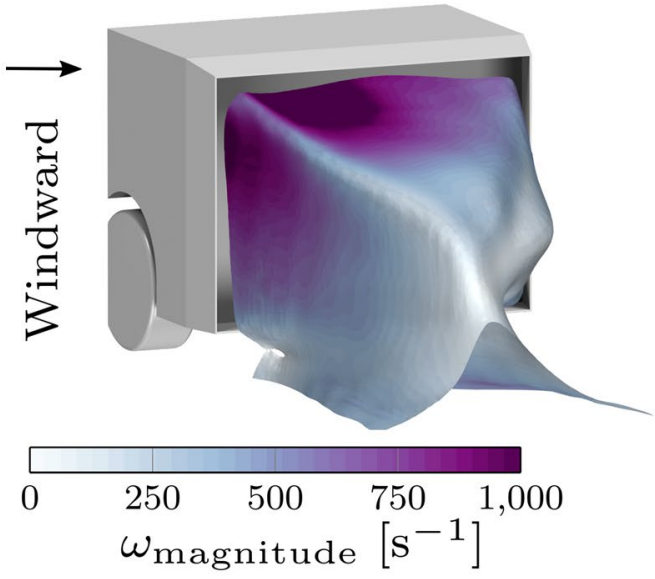

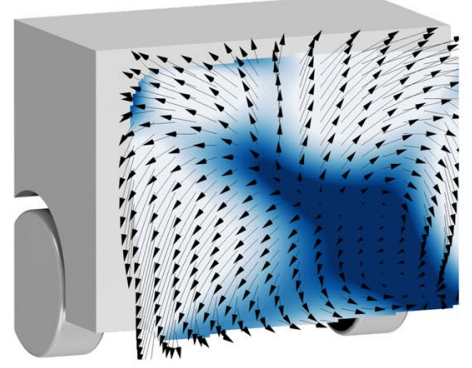

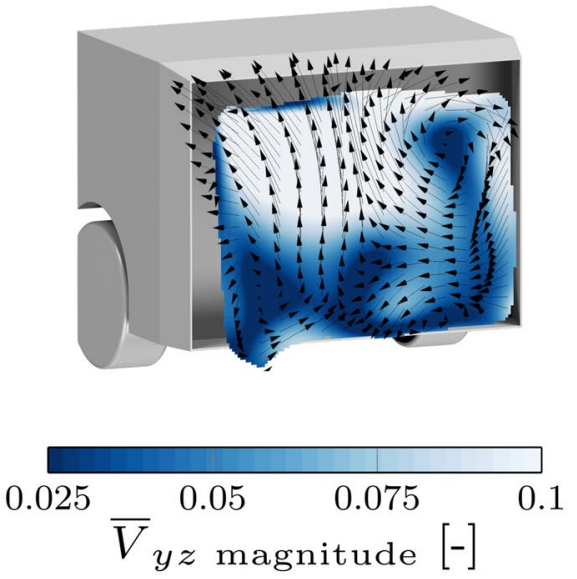

\section{Introduction}

There is a demand for more energy-efficient vehicles as greenhouse gas emissions and air quality regulations have become more strict. One of the key performance indicators of energy efficiency is the aerodynamic drag. The aerodynamic drag accounts for more than a quarter of the traction energy required based on the WLTP test cycle (WLTP is the World Light Vehicle Test protocol that is widely used in the certification of vehicle emissions) Pavlovic et al. (2016).

Road vehicle flow fields are characterised by large unsteady wakes, and the drag is therefore dominated by pressure drag that accounts for approximately for approximately $90 \%$ of the total drag Schuetz (2015). As such, much focus has been placed on increasing the base pressure of the vehicle.
Although bluff body wakes continue to be an extensively researched topic, most of the investigations are performed in ideal conditions without yaw. Tunay et al. (2018) and Josefsson et al. (2018) have shown that the effects from wind, traffic and the vehicle movement can all influence the aerodynamic drag. There are still a few studies of vehicle aerodynamics at yaw, for example (Varney et al. 2018a; Windsor 2014; Howell 2015; Pfeiffer and King 2018; Volpe et al. 2015; Li et al. 2019; Bonnavion and Cadot 2018; Garcia de la Cruz et al. 2017; Lorite-Díez et al. 2020a). Gaylard et al. (2014) showed that the drag reduction benefit of front-wheel deflectors was halved when tested with realistic on-road turbulence compared to a low turbulence environment. The improvements close to the front wheel deflectors were maintained; however, the downstream improvements were reduced under turbulent conditions. Howell et al. (2018) showed that vehicles with similar drag coefficients at $0^{\circ}$ yaw can have large differences in drag in a crosswind. 
In a study of 51 passenger vehicles, Windsor (2014) found that the sensitivity to yaw was generally higher for vehicles with low drag at $0^{\circ}$-yaw. Howell et al. (2018) addressed the need to account for the operating conditions by introducing a driving cycle equivalent drag measure which takes into account the distribution of wind velocities and vehicle velocities to calculate a drag figure that is representative of a full driving cycle. Howell et al. (2018) also introduced a method for estimating the cycle averaged drag using just four representative yaw angles $(0,5,10$ and 15 degrees), to give an indication of the vehicle's aerodynamic performance in representative wind conditions at a lower cost. It is noted that the measurements are obtained from wind tunnel tests, so do not consider unsteady effects, for example, associated with traffic and the environment.

Drag reduction mechanisms have been characterised in the literature using different qualitative and quantitative indicators of the wake such as size, shape, spectral information, modal information, closure point location, kinetic energy, turbulent kinetic energy, recirculation angles and vorticity distribution (Barros et al. 2016; Duell and George 1999; Pavia et al. 2020a; Bonitz 2018; Urquhart et al. 2018). However, the wake information available is often limited to one or a few planes and typically does not encompass a broader range of operating conditions. Changes to the base flow can influence several aspects of the wake, and determining all planes of interest a priori is not possible due to the highly 3-dimensional nature of the wake, especially at yaw. Tomographic Particle Image Velocimetry (TPIV) is used in this work to sample the complete wake. The initial setup time and additional cost for TPIV can be greater than planar PIV; however, the setup and testing of several planar PIV planes can become prohibitively expensive as the number of planes required increases.

Another aspect, further complicating drag reduction in the wake, is the trade-off between drag on rearwards facing tapered surfaces with attached flow and the surfaces which are in separated flow Ahmed et al. (1984). This effect has been investigated by Haffner et al. (2020a) on a simplified vehicle where the pressure drag from the Coanda effect on small curved surfaces with pulsed jets was considered. Mariotti et al. (2017) investigated transverse grooves to delay separation on a boat-tailed axisymmetric body where the delay in flow separation increased the base pressure while decreasing the pressure on the curved surfaces.

There are several devices and methods to increase the pressure recovery in the wake by altering the base flow, improving wake balance, reducing wake size, increasing bubble length, reducing the shear layer mixing etc. One example is a rearward-facing cavity.

Applying a cavity to the base of the geometry, by extending the perimeter, is effective at $0^{\circ}$ yaw (Evrard et al. 2016; Duell and George 1993; Bonnavion et al. 2019; Lucas et al.
2017), with as much as $11 \%$ reduction in drag Duell and George (1993). Cavities are often tapered to reduce the wake size and further increase the drag reduction. Tapering without the use of a cavity is also an effective drag reduction technique, with up to 20\% reduction in drag (Ahmed et al. 1984; Varney et al. 2018a). The combination of a cavity and tapering has been seen to further improve the drag reduction Cooper (1985).

This work investigates the wake of a straight cavity and a tapered cavity optimised to minimise the driving cycle averaged drag coefficient on a generic square-back vehicle. For this, forces and base pressure measurements and full wake Tomographic Particle Image Velocimetry are used to explain the drag reduction mechanisms at yaw.

\section{Methodology}

\subsection{Test facility}

Experimental work was conducted in the Loughborough University Large Wind Tunnel, as shown in Fig. 1. The test section is $1.92 \mathrm{~m} \times 1.3 \mathrm{~m}$ with $0.2 \mathrm{~m}$ corner fillets and is $3.6 \mathrm{~m}$ long. The maximum achievable velocity in the test section is $45 \mathrm{~m} / \mathrm{s}$ with a freestream turbulence intensity of $0.2 \%$ and a flow uniformity of $\pm 0.4 \%$ Johl (2010).

The model is mounted to the wind tunnel balance with four $8 \mathrm{~mm}$ pins which are located between the wheels to reduce the impact on the overall flow. A debossed floor pad is located underneath each wheel to prevent grounding of the wheels which are tangential to the tunnel floor Pavia and Passmore (2018). The ride height is constant at $50 \mathrm{~mm}$, or 0.17 times the base height. The pitch and roll angle of the geometry is set to be within $0^{\circ} \pm 0.1^{\circ}$. The $0^{\circ}$-yaw angle is found by sampling the base pressures in $0.1^{\circ}$-yaw increments where the angle with the lowest lateral centre of pressure deviation is selected.

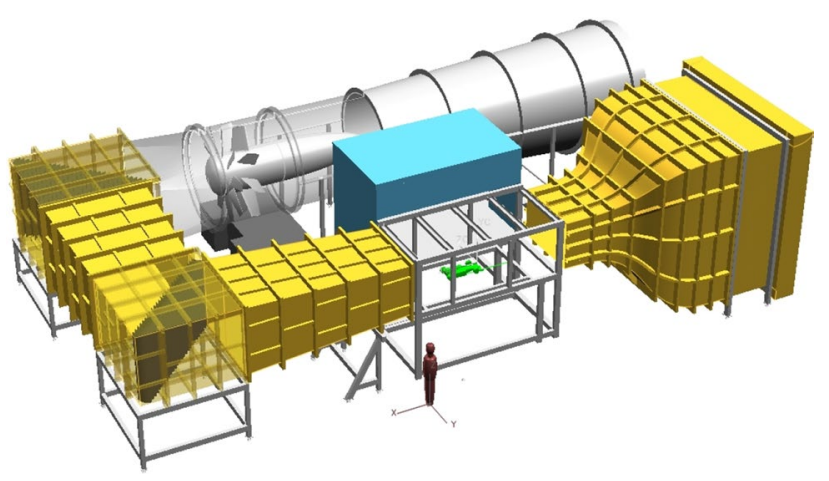

Fig. 1 Loughborough University Large Wind Tunnel Johl (2010) 
Only negative yaw angles were tested experimentally to avoid obscuring part of the wake from the cameras when performing PIV. The force and pressure measurements were also measured at negative yaw angles to stay consistent with the PIV results. The results are expected to be the same for both negative and positive yaw angles since the geometry is symmetric. Because of this, all results are mirrored to reflect a positive yaw angle with the nose pointing right following the SAE standard J1594 (J1594 1954). In the presented results, the wind moves from left to right in the vehicle driving direction when the model is yawed.

\subsection{Geometry}

The test object is the Windsor body which is a simplified automotive test case. The Windsor body is a wellresearched model used to study fundamental flow physics in road vehicles (Pavia and Passmore 2018; Pavia et al. 2018; Perry et al. 2016; Pavia et al. 2020b; Favre and Efraimsson 2011; Howell et al. 2013; Varney et al. 2018b). The model used in this paper is a modification with wheels to create a more representative flow field of a production vehicle, applied by Pavia and Passmore (2018), as shown in Fig. 2a. The wheels have a diameter of $150 \mathrm{~mm}$, a width of $55 \mathrm{~mm}$ and a corner radius of $5 \mathrm{~mm}$. The wheels are mounted flush with the side of the geometry. Pavia and Passmore (2018) and Wang et al. (2019) investigated the effects of wheel rotation and found qualitatively similar wakes between stationary and rotating wheels. The wheels are mounted to the model and are stationary in this work. The scale model is approximately nicefrac 14 of a production vehicle with a blockage ratio of $4.7 \%$.

\subsubsection{Cavity}

Two cavities were tested in this work, a straight cavity with $3 \mathrm{~mm}$ thick plates protruding from the base perimeter and a tapered cavity with the same wall thickness, as shown in Fig. 2. The cavities are 50mm long, or 0.173 of the base height, measured perpendicular from the base of the vehicle to the trailing edge of the cavity. This length of cavity has been investigated previously by Varney (2020) and was chosen as a compromise between practicality and drag reduction. Longer cavities are known to be able to reduce drag further and stabilise the wake Evrard et al. (2016); Varney (2020); Sterken et al. (2014). The cavities are compared to the square-back without a cavity as well as an additional configuration where the tapered cavity was filled to test the effects of tapering alone, as shown in Fig. 2d. The filled tapered cavity does not have base pressure measurements.

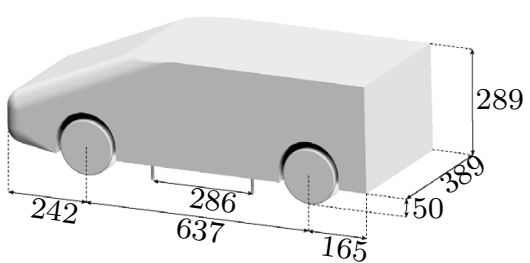

(a) Square-back.

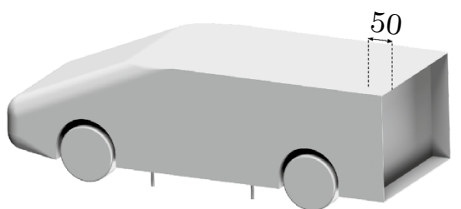

(b) Straight cavity.

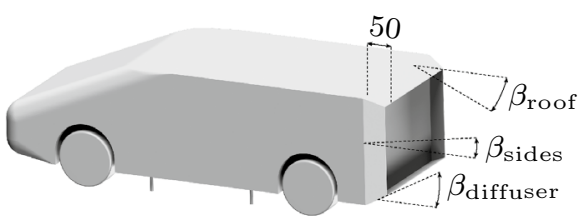

(c) Tapered cavity.

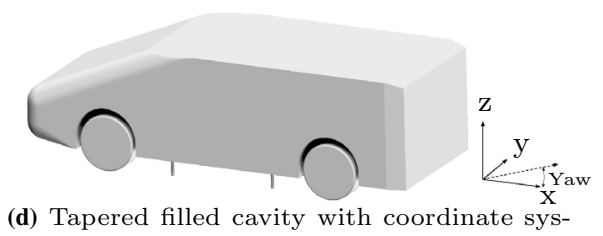
tem.

Fig. 2 Windsor geometry with wheels and coordinate system. Measurements are given in $\mathrm{mm}$

\subsubsection{Cavity optimisation}

The tapered cavity angles were optimised using steady-state CFD simulations. A driving cycle equivalent drag value, $C_{\text {DwC }}$, developed by Howell et al. (2018) was used as the objective function which considers the wind distribution and driving cycle to arrive at an equivalent drag value. The variant used is an engineering estimate of the full cycle averaged drag and has been modified by Varney et al. (2018a) to omit the small contribution of the $15^{\circ}$ yaw term in the original formulation. The relative weighting of each yaw angle follows no physical meaning and is found using curve fitting.

$C_{\text {DWC }}=0.53 C_{\mathrm{D}} 0^{\circ}$ yaw $+0.345 C_{\mathrm{D}} 5^{\circ}$ yaw $+0.13 C_{\mathrm{D}} 10^{\circ}$ yaw

The simulation method is based on the setup used in (Urquhart et al. 2018). The turbulence model was changed to a steady-state low $y^{+}<1 k$ - $\omega$ SST turbulence model. A steady-state approach was needed to reduce the optimisation cost as each objective function requires the simulation to be run at three yaw angles, which were re-meshed between each run as the model was yawed. The computational domain 
included the physical wind tunnel to capture the boundary layer growth from the inlet to the test section, as well as simulate the correct blockage ratio. At the model centreline, the boundary layer is approximately $60 \mathrm{~mm}$ for an empty tunnel.

Figure 3 shows a mesh study of the square-back configuration from $15 \times 10^{6}$ to $150 \times 10^{6}$ cells where the predicted drag change between $0^{\circ}$ - and $10^{\circ}$-yaw is shown.

This is compared to the cube root of the number of cells and is closely related to the edge length of each cell in a hexahedral mesh. The increasing trend in the drag delta is due to the drag prediction at $10^{\circ}$-yaw increasing as the cell count is increased. As the mesh is refined, the steady-state simulations exhibit increasingly unsteady behaviour linked to the unsteady nature of the problem, particularly prevalent at yaw. The mesh with approximately $90 \times 10^{6}$ cells was selected as a compromise between simulation accuracy and cost.

Unsteady simulations were performed to study the prediction accuracy using a higher fidelity but more expensive simulation method. The same mesh is used with a Improved Delayed Detached Eddy Simulation (IDDES) $k$ - $\omega$ SST turbulence model Shur et al. (2008). A timestep of $2.0 \times 10^{-4} \mathrm{~s}$ was used with an averaging time of $2 \mathrm{~s}$ after reaching fully developed flow. Figure 4 shows different simulation methods compared to wind tunnel experiments for the square-back.

The prediction in both the trend and absolutes improve with the unsteady method, which is an expected result due to the large unsteady wake. The absolute prediction difference is larger than expected compared to full-scale testing (Urquhart et al. 2018). These differences are thought to be due to geometric factors that were not considered such as leakages, gaps in the model, surface roughness and mounting hardware which are exaggerated at model scale. However, these factors are not expected to influence the changes from configurations or the predicted optimum design. The predicted drag change at $10^{\circ}$-yaw for the steady method is under-predicted due to the unsteady nature of the problem. This is, however, a lesser concern due to the lower weighting of the $10^{\circ}$-yaw drag value in the cycle averaged drag. The unsteady method is an order of magnitude more expensive

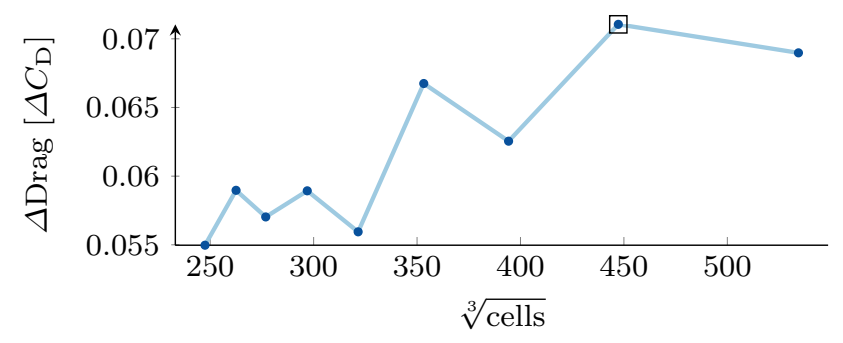

Fig. 3 Mesh study of the square-back geometry where $\Delta C_{\mathrm{D}}$ is the difference in drag between $0 \circ-$ and $10^{\circ}$-yaw. The selected mesh is marked with a square

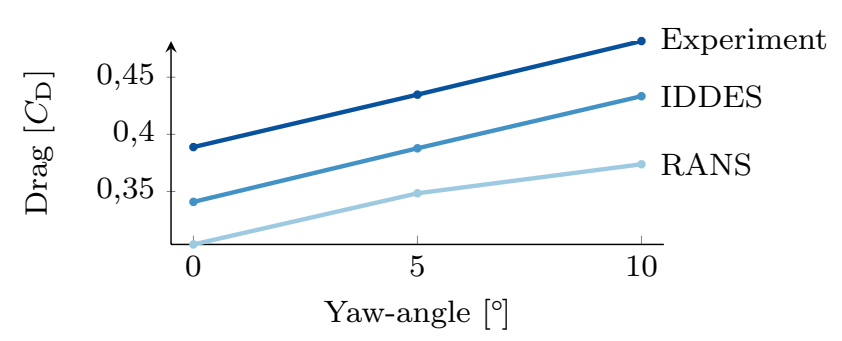

Fig. 4 RANS, IDDES and wind tunnel test results of the square-back geometry

than the steady-state simulations, and because of this, the steady-state approach was chosen.

The angles of the cavity were optimised using an opensource surrogate model-based algorithm using Radial Basis Function (RBF) interpolation (Urquhart et al. 2020a). An ensemble of 10 surrogate models was used to improve the optimisation convergence. The tested designs were alternated between the design with the lowest median prediction of the 10 surrogates and the largest standard deviation between the surrogates. This ensures that the existing knowledge is exploited while exploring the design space where the surrogate model accuracy is lower. The entire ensemble was retrained between iterations.

The surrogate model was built from an initial design of experiments of 15 samples using a Latin Hypercube (LHC) which divides each design parameter into equally sized intervals. The same value for a given design parameter can only occur once. The placement of each sample is optimised to reduce clustering within the sampling plan following the work by Bates et al. (2004). More details on the optimisation process can be found in Urquhart et al. (2020a).

The tapering was constrained to a maximum of $25^{\circ}$. Figure 5 shows the optimisation history of the designs. A total of 42 objective function calls, or 126 simulations, were run.

The optimisation routine was stopped when it was deemed that no further significant reduction in drag could be found in close vicinity to the already tested designs. The best found design had a roof angle of $12.6 \circ$, diffuser

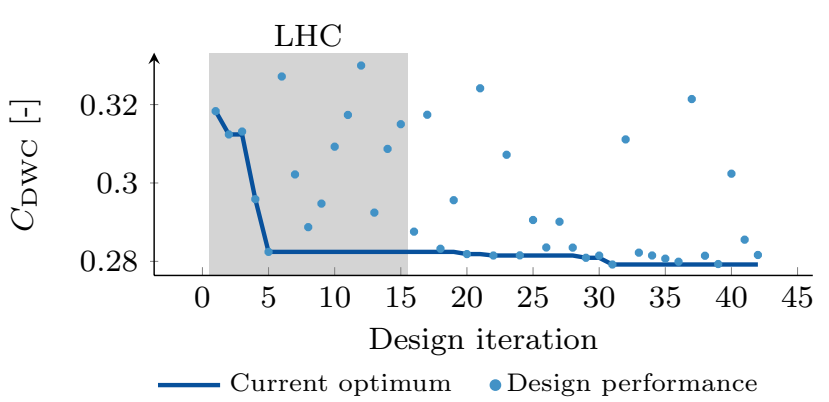

Fig. 5 Cavity optimisation history 


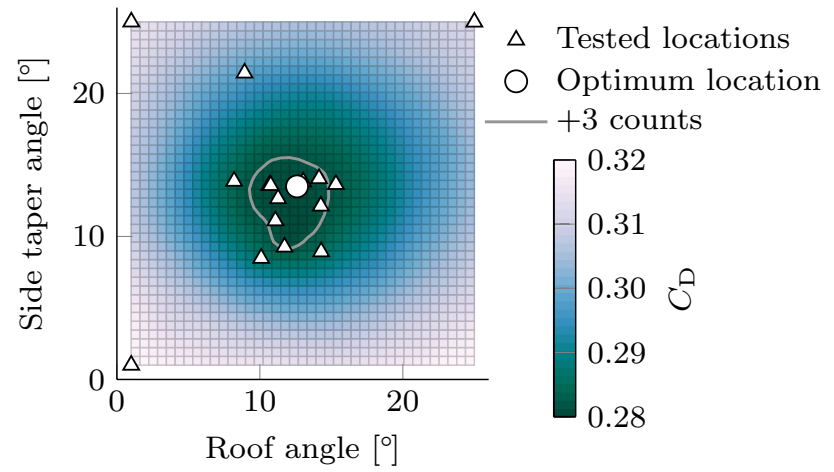

Fig. 6 Sensitivity to roof- and side taper-angle for the minimum diffuser angle. Tested design locations include all designs with a diffuser angle below $2^{\circ}$

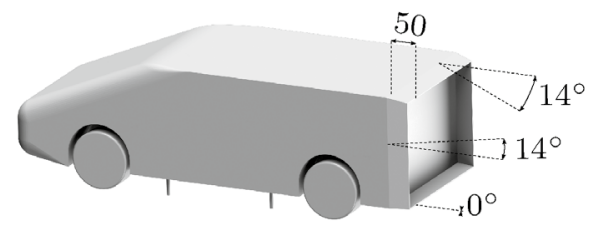

(a) Optimised tapered cavity.

Fig. 7 Windsor geometry with wheels and tapered cavity. Measurements are given in $\mathrm{mm}$

angle of $1 \circ$ and side tapering of $13.5 \circ$. Several of the tested designs with low drag at 0o-yaw had large increases in drag at yaw. For example, a design with identical $0^{\circ}$-yaw drag to the optimum was found with a 5\% larger cycle averaged drag. This highlights the importance of considering a wider range of operating conditions when optimising a vehicle.

The design parameter sensitivity was tested using the surrogate model to predict the cycle-averaged drag in the entire design space and is shown in Fig. 6. The design area within $10^{\circ}$ to $15^{\circ}$ in both side tapering and roof tapering angle is within approximately 3 counts, or $0.003 C_{\mathrm{D}}$ of the optimum location indicating low sensitivity near the optimum.

Based on the sensitivity analysis, a design with $14^{\circ}$ side- and roof-tapering with a $0^{\circ}$ diffuser was manufactured with a predicted improvement of $0.051 C_{\text {DWC }}$ (often referred to as 51 counts) and approximately $15 \%$ over the square-back geometry. The manufactured cavity design is shown in Fig. 7. The slightly larger side and roof tapering was selected to allow easier addition of surfaces to the outside of the cavity if needed. These angles are similar to those reported in previous work on cavities in crosswind conditions (Sterken et al. 2014).

\subsection{Force measurements}

All force and pressure measurements were taken with a freestream velocity, $v_{\infty}$, of $40 \mathrm{~m} / \mathrm{s}$ measured with a pitotstatic tube located $1.15 \mathrm{~m}$ upstream of the model leading edge. This results in a Reynolds number, based on the square root of the frontal area of the vehicle, of $R e_{\sqrt{\mathrm{A}}}=9.6 \times 10^{5}$. The balance data were sampled at $300 \mathrm{~Hz}$ for 300 s with an arithmetic mean taken in post-processing. The balance is rotated with the model as the model is yawed and the coordinate system remains fixed with the vehicle.

The coefficient calculations are presented in Equations (2-5), where $A$ is the model's projected frontal area $\left(\mathrm{m}^{2}\right)$, $\rho$ is the calculated air density $\left(\mathrm{kg} / \mathrm{m}^{3}\right), v_{\infty}$ is the freestream velocity as measured at the pitot tube upstream $(\mathrm{m} / \mathrm{s})$ and $L_{w}$ is the length of the wheelbase $(m)$. The coefficients are defined as

$C_{\text {Force }}=\frac{\text { Force }}{\frac{1}{2} \rho A v_{\infty}^{2}}$
$C_{\text {Moment }}=\frac{\text { Moment }}{\frac{1}{2} \rho L_{w} A v_{\infty}^{2}}$

$C_{\mathrm{RL}}=\frac{1}{2} C_{\mathrm{L}}-C_{\mathrm{M}, \mathrm{Pitch}}$

$C_{\mathrm{FL}}=\frac{1}{2} C_{\mathrm{L}}+C_{\mathrm{M}, \mathrm{Pitch}}$

where the subscript RL denotes rear lift and FL denotes front lift at the wheel contact patch. The vehicle yaw moment is defined using the right-hand rule along the axis of lift, i.e. a positive yaw moment pulls the vehicle nose left.

The repeatability between tests was estimated by running the same configuration on different days, resulting in a total of 7 unique samples. From these samples, the 95\% confidence interval was calculated to \pm 1 counts at $0^{\circ}$ yaw, \pm 2 at $5^{\circ}$ yaw and \pm 2.5 at $10^{\circ}$ yaw.

\subsection{Pressure measurements}

The surface static pressure measurements were recorded for $300 \mathrm{~s}$ at $260 \mathrm{~Hz}$ using a differential pressure scanner with 64 channels via $500 \mathrm{~mm}$ long smoothbore silicone tubing. Wood (2015) showed that the tubing length can change the amplitude and phase of the pressure signal in a similar setup. His results showed an amplification by $10 \%$ with a phase shift of $3 \%$ at a Strouhal number of 0.25 where the changes were successively smaller at lower frequencies. Therefore, no correction was applied to the data in 


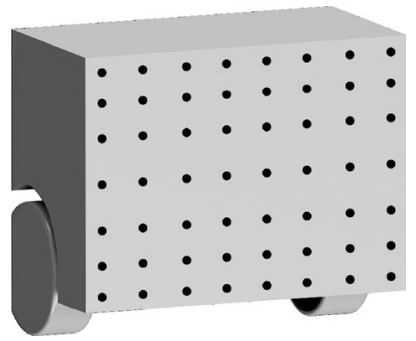

Fig. 8 Distribution of the $7 \times 8$ wall static pressure measurement locations

this work as frequencies less than $0.25 \mathrm{St}$ are of interest. The scanner was located inside the model with all necessary cabling exiting behind the forward mounting pins to reduce the impact on the flow. A time correction is applied to the data which time-aligns each of the 64 channels. The implementation of this is well documented by Wood (2015). The distribution of pressure tappings on the base is presented in Fig. 8.

To calculate the contribution of the base of the model to the overall drag, $\left(C_{\mathrm{DB}}\right)$, the pressure coefficient for each tapping location is integrated using Equation (6) and by associating an incremental area with each tapping and the surface normal. Using the same analysis as the force measurements, the repeatability was $\pm 0.002 C_{\mathrm{DB}}$. The resulting value is representative of the base drag, but as neither the internal or external walls of the tapered cavity are pressure tapped, it does not accurately represent the total contribution to the rear pressure drag.

$C_{\mathrm{DB}}=\frac{1}{A} \int_{A_{b}} C_{\mathrm{P}} \cdot d A_{b} \cdot n_{x}$

Similarly, the moment on the base, $\left(C_{\mathrm{MB}}\right)$, is calculated by adding the contribution from each pressure measurement around the centre of the base. The lateral and vertical base moment is normalised by the width and the height of the base, respectively.

Two-point correlation is used to evaluate the temporal correlation of one base pressure tap to all of the pressure taps on the base. The two-point correlation for a flow variable, $\xi$, and two of the pressure taps is defined as

$C_{\xi}\left(x_{A}, x_{B}\right)=\overline{\xi^{\prime}\left(x_{A}\right) \xi^{\prime}\left(x_{B}\right)}$

where $x_{A}$ and $x_{B}$ are the spatial coordinates of interest and $\xi^{\prime}$ is a vector of the fluctuating flow variable $\xi$. The overline denotes time averaging throughout this work. The correlation is large and positive for two correlated signals in phase, large and negative for two correlated signals $180^{\circ}$ out of phase and small for uncorrelated signals. A variant of the correlation is also used which is normalised by the product of the RMS of each correlated signal resulting in a selfcorrelated signal becoming 1 .

\subsection{Tomographic particle image velocimetry}

The flow field of the near wake was captured using Tomographic Particle Image Velocimetry (TPIV) and processed using commercially available hardware and software (DaVis ${ }^{\circledR}{ }^{8.4}$ ) from LaVision ${ }^{\circledR}$. For each measurement, 1000 image pairs were taken at a frequency of $5 \mathrm{~Hz}$, resulting in 200s of data, which has been shown to be sufficiently large (Pavia et al. 2019, 2020b; Perry et al. 2016). The resulting statistical error from the number of image pairs taken for the $95 \%$ confidence interval of the mean velocity is on the order of $0.3 \%$ in the freestream and $5 \%$ in the highly turbulent shear layers (Pavia et al. 2020b; Perry et al. 2016).

The volume captured in the tomographic PIV was $550 \mathrm{~mm} \times 480 \mathrm{~mm} \times 380 \mathrm{~mm}$ in the $x, y$ and $z$-direction, chosen to capture the full width and height of the wake and the wake closure point based on previous work (Pavia et al. 2020b; Perry et al. 2016; Pavia and Passmore 2018).

Four cameras, two Imager Pro X 4M cameras (4MP) and two sCMOS cameras (5MP), were arranged (Fig. 9) such that the inclusive angle between them was $60^{\circ}$, leading to a reconstruction quality of approximately 0.95 (Scarano 2013). Each camera was equipped with a $35 \mathrm{~mm}$ focal length Nikon ${ }^{\circledR}$ lens, and to satisfy the Scheimpflug condition the f-stop was set to $f^{\#}=8$ for all the cameras. This was chosen as a compromise between depth of field and capturing sufficient light. The resolution of all images was reduced to $25 \%$ of their original resolution in post-processing because of memory limitations during processing.

Initial calibration was performed using a $2 \mathrm{D}$ calibration plate located in five positions within the field of view: streamwise centreline, the two streamwise outer edges and two diagonals. The final calibration was done by performing several self-calibration iterations. This process results in a map of the position of a region of the image to a region of

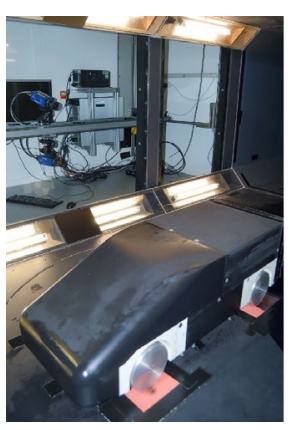

(a) Camera position and orientation relative to the model

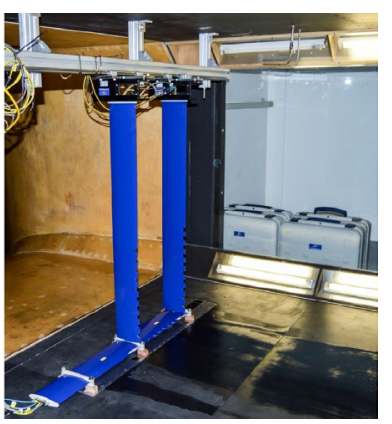

(b) Seeding set up
Fig. 9 Tomographic set up in the working section 
the volume for each of the cameras, resulting in an RMS error, between a true rectilinear grid and reconstructed one, of less than 0.006 pixels.

Illumination of the measurement volume was provided with a $200 \mathrm{~mJ} \mathrm{Nd}$ :YAG double-pulse laser from Litron ${ }^{\circledR}$. The laser beam was passed through a volume optic, chosen to ensure that $\approx 60 \%$ of the peak power from the Gaussian distribution covered the required measurement volume of interest.

The much-reduced light intensity, compared to planar PIV, means that it is necessary to use large seeding particles, as particle reflectance is related to the cube of the particle size (Adrian and Yao 1985). The flow was seeded using $300 \mu \mathrm{m}$ diameter Helium Filled Soap Bubbles (HFSB), introduced using a commercial seeder and three seeding rakes positioned at the start of the working section (Fig. 9b). Each rake, consisting of 10 nozzles, produces 400,000 bubbles per second, with one rake positioned horizontally to seed the boundary layer and underbody flow and two to seed the bulk flow. The use of HFSB is reported widely in the literature (Perry et al. 2016; Caridi et al. 2016; Kühn et al. 2011; Pavia et al. 2019, 2020b), and the particles have been shown to follow the flow similarly to other seeding mediums (Scarano et al. 2015). The location of the rakes causes a small increase in the freestream turbulence, but this is unavoidable to ensure even seeding of the shear layers. The seeding was continuous throughout the testing due to the open return design of the wind tunnel.

The resulting seeding density is insufficient to generate high quality PIV data at $40 \mathrm{~m} / \mathrm{s}$. The PIV was therefore conducted at $30 \mathrm{~m} / \mathrm{s}$ to maintain a particle seeding density of about 0.02 particles per pixel at $25 \%$ resolution, required to reduce the particle reconstruction error (Elsinga et al. 2011). This is well above the minimum required for a Reynolds insensitive flow field where drag, base drag and surface pressures around this specific model were investigated Varney (2020). The force and base pressure measurements were conducted at $40 \mathrm{~m} / \mathrm{s}$ to maximise the resolution from the balance and pressure measurement systems.

To reduce reconstruction error, a series of pre-processing steps were applied to the images to increase the signal-tonoise ratio between the particles and the background. The tomographic reconstruction uses the FastMART (Fast Multiplicative Algebraic Reconstruction Technique) as implemented by LaVision ${ }^{\circledR}$ based on the work of Atkinson and Soria (2009). This technique reconstructs the particles in $3 \mathrm{D}$ space from a series of $2 \mathrm{D}$ images and is performed iteratively, six times, to reduce the number of false-positive reconstructed (ghost) particles present in the data (Scarano 2013; Elsinga et al. 2006). Ghost particles bias the volume to the mean flow of the entire volume (Elsinga et al. 2011), so minimising them is beneficial for data quality.

The vectors were calculated using a direct correlation method with multiple passes. The initial pass used a
$256 \times 256 \times 256$ pixel window with a large $(\times 8)$ volume bin and ended with a $64 \times 64 \times 64$ pixel window with no volume binning, resulting in $48 \times 50 \times 37$ vectors in the $x, y$ and $z$-direction with a spacing of $12 \mathrm{~mm}$ in each direction.

\section{Results and Discussion}

Results are first presented for the zero yaw condition followed by the yawed results where primarily the $5^{\circ}$-yaw results are discussed. All the flow fields and pressure measurements presented in this section are generated from the wind tunnel experiments.

\section{$3.10^{\circ}$-yaw}

The square-back and cavity geometries were investigated for lateral and vertical symmetry breaking modes as this has previously been reported in the literature for similar models (Volpe et al. 2015; Bonnavion and Cadot 2018; Barros et al. 2016; Bonnavion et al. 2019; Pavia and Passmore 2018; Pavia et al. 2018; Perry et al. 2016; Grandemange et al. 2013; Li et al. 2016) and is linked to the vehicle drag. The probability density function for a laterally located pressure tap as well as the vertical pressure gradient showed no signs of switching between symmetry breaking modes for any of the geometries. This is aligned with previous studies for this model when wheels, rear tapering or cavities at, or longer than, $0.173 \mathrm{H}$ are used where the lateral symmetry breaking modes revert to the mean flow Perry (2016); Varney (2020). Bonnavion and Cadot (2018) investigated two flatbacked Ahmed bodies with differing rectangular base aspect ratios where vertical and lateral instabilities were found to be dependent on factors such as ground clearance or vehicle pitch angle. Their results show that the symmetry breaking mode has a modulus and phase with a dominant direction, lateral or vertical, where the other direction instead exhibits a flow without instabilities. This is in line with the results observed here where the wheels cause the wake to be locked into one state, hindering lateral or vertical bi-stability.

The force coefficients for the four configurations, squareback, straight cavity, tapered cavity and the filled tapered cavity are given in Table 1 . A straight cavity provides a $7.5+-0.4 \%$ reduction in drag compared to the square-back.

Table 1 Aerodynamic force coefficients at $0^{\circ}$-yaw

\begin{tabular}{lllll}
\hline Configuration. & $C_{\mathrm{D}}$ & $C_{\mathrm{FL}}$ & $C_{\mathrm{RL}}$ & $C_{\mathrm{DB}}$ \\
\hline Square-back & 0.389 & 0.142 & 0.002 & 0.291 \\
Straight cavity & 0.360 & 0.140 & -0.007 & 0.259 \\
Tapered cavity & 0.320 & 0.121 & 0.166 & 0.200 \\
Filled tap. cav. & 0.335 & 0.124 & 0.166 & - \\
\hline
\end{tabular}


Fig. 10 Time-averaged centreline velocity magnitude normalised by the freestream velocity. The red line is a qualitative addition to indicate the return flow direction and is placed manually in the region of large backflow velocity

In total, a $17.6+-0.4 \%$ reduction in drag is achieved with the tapered cavity compared to the square-back. The cavity on the tapered geometry reduces drag by $4.5+-0.4 \%$, compared to the filled taper, which is a smaller relative improvement compared to the straight cavity. While the improvement is smaller, there is still a significant reduction in drag when combining tapering with a cavity. It is worth noting that the straight cavity is longer than the square-back which can exaggerate the performance increase. The front lift decrease from the taper can be explained by a local decrease in pressure on the outside of cavity as well as a global change in front stagnation point where it is moved upward by the taper, reducing front lift. The tapered cavity adds a significant amount of rear lift which can impact vehicle high-speed stability (Howell and Le Good 1999).

The tapering reduces the wake volume, both cross-sectional area and length, as well as reduces the velocity of the flow from the underbody, as shown in Fig. 10. The in the figure is placed manually in the region of large backflow velocity along the flow direction in this work. An alternative is to place the line between the point of maximum backflow in the wake and maximum pressure on the base; however, this was not done since the filled tapered cavity lacks base pressure information.

The square-back and straight cavity is upwash dominated, whereas the tapered cavity produces a downwash dominated wake. Filling the tapered cavity adds more upwash to the wake, but the length is reduced and the vertical height of the wake is increased. Figure 11a-11b shows the expected base pressure distributions of an upwash dominated wake and Fig. 11c the expected distribution for downwash.

Figure 12 shows the turbulent kinetic energy for the tapered cavity and the filled taper. The turbulent kinetic energy in the shear layer at the roof is lower for the configuration with a cavity. The reduction in wake length is associated with higher levels of unsteadiness and mixing in the shear layers due to increased entrainment of high energy flow into the wake, increasing drag, which is also seen here (Barros et al. 2016). Evrard et al. (2016) applied a cavity to a simplified bluff body and observed a similar stabilisation of the roof shear layer. Haffner et al. (2020b) ascribe this to a reduction in the interaction between opposing shear layers which reduces the turbulent fluctuations across the shear layer while reducing the engulfment of high energy flow into the wake. The PIV data show that the cavity reduces the turbulent fluctuations across the shear layers as well as re-aligning the recirculating flow in the wake with the freestream direction before mixing at the trailing edge of
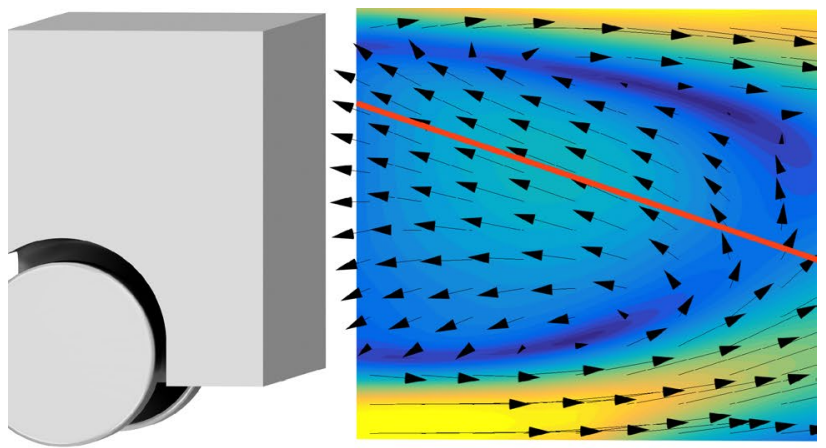

(a) Square-back

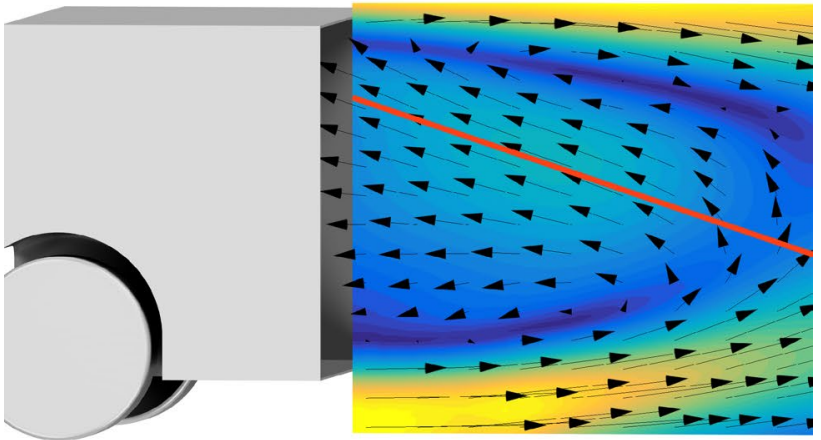

(b) Straight cavity

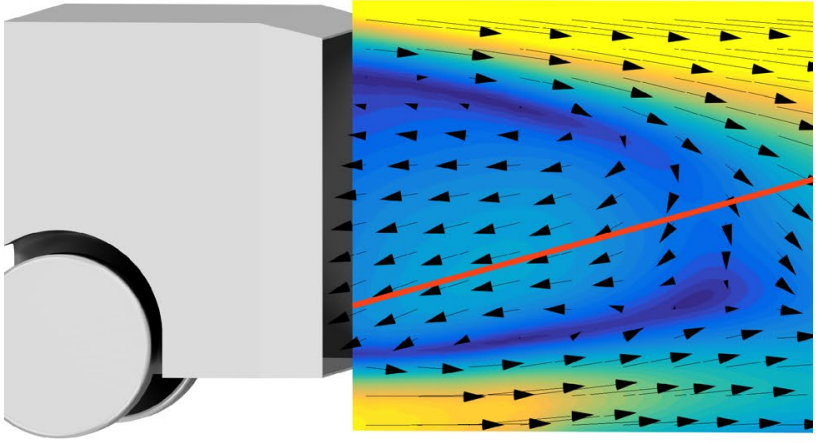

(c) Tapered cavity

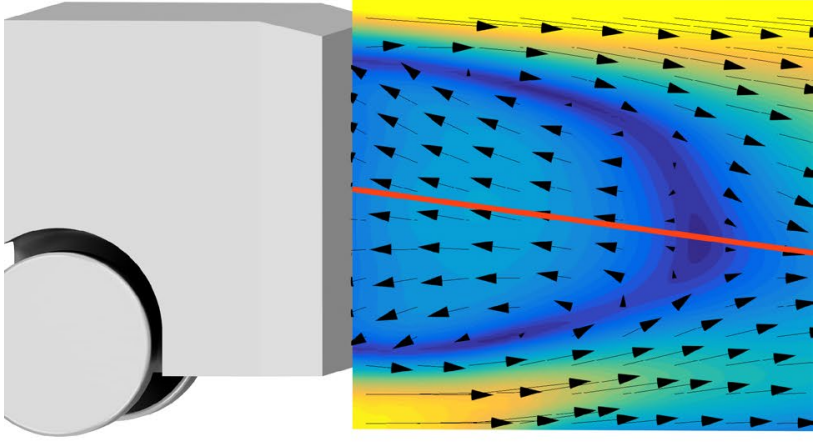

(d) Tapered filled cavity

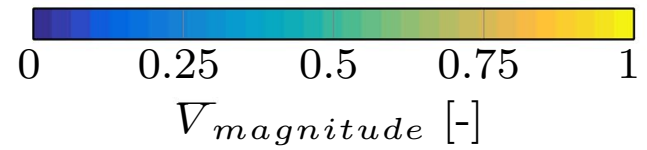




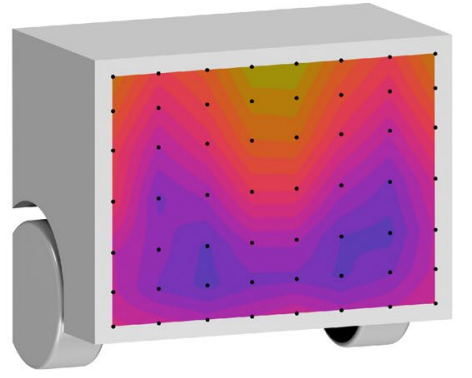

(a) Square-back

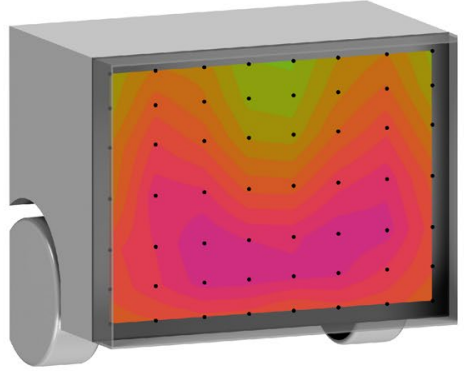

(b) Straight cavity

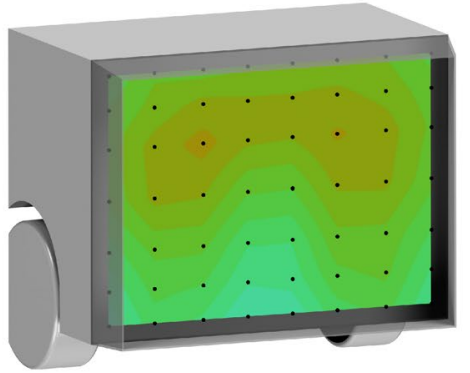

(c) Tapered cavity

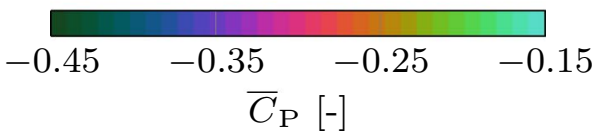

Fig. 11 Time-averaged base pressure at $0^{\circ}$-yaw of the square-back, straight cavity and tapered cavity. The pressure tap locations are marked in black

the cavity. This reduces the relative velocity difference of the flow at the shear layer, stabilising it. Lorite-Déez et al. (2020b) applied steady blowing to the rear edges of an Ahmed body and observed a similar effect depending on blowing velocity where the side that was blown had reduced turbulent fluctuations in the shear layers. These results are consistent with Grandemange (2013) who showed that reducing the spanwise turbulent fluctuations across a mixing layer increases the pressure on the low velocity side. Reducing the spanwise turbulent fluctuations for a bluff body would, in that case, reduce drag. (Han et al. 2013) applied unsteady flow control to the trailing edge of a simplified vehicle model which reduced the shear layer instabilities,

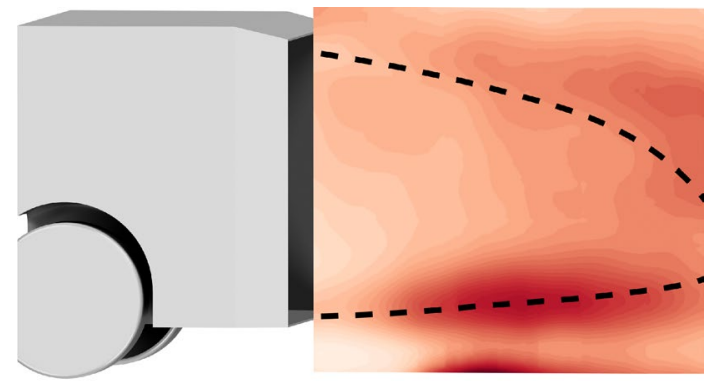

(a) Tapered cavity

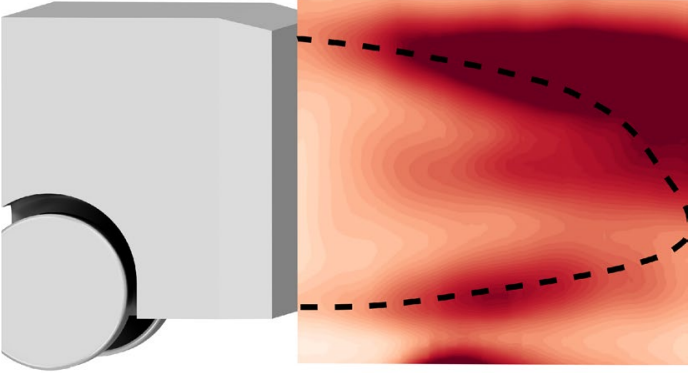

(b) Tapered filled cavity

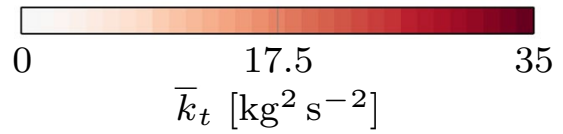

Fig. 12 Time-averaged turbulent kinetic energy at the centreline for the tapered cavity and the filled tapered cavity. The line represents the wake where the longitudinal velocity is $25 \%$ of the freestream velocity

reducing the turbulent kinetic energy along the shear layers. This elongated the wake, reducing drag. The centreline flow, as shown in Fig. 10, shows an increase in the length to height ratio, or reduced curvature, for the configurations with a cavity compared to the counterparts without a cavity, which is mainly due to a reduction in the height of the wake. This indicates that the pressure difference between the inside of the wake and the freestream is reduced by the cavity as the faster wake closure increases the centripetal acceleration Grandemange (2013). The base pressure fluctuations are reduced with the addition of a cavity and further reduced with the taper, as shown in Fig. 13. The areas of high base pressure fluctuation are located near the impingement location based on the centreline flow, as shown in Fig. 10, and the base pressures, as shown in Fig. 11.

Figure 14 shows the base coefficient of moment around the horizontal and vertical midpoint to analyse the strength of the fluctuating wake on the base. For the tapered cavity, the base moment coefficient around the horizontal axis is positive, or pitching the nose up. For the square-back and the straight cavity the $C_{\mathrm{MB}, y}$ is negative, indicative of the upwash dominated wake. A narrower band of $C_{\mathrm{MB}, y}$ is observed for the tapered cavity with a peak closer to 0 .

Around the vertical axis, $C_{\mathrm{MB}, z}$ is reduced by the cavities, indicating that the lateral flapping strength is reduced, 


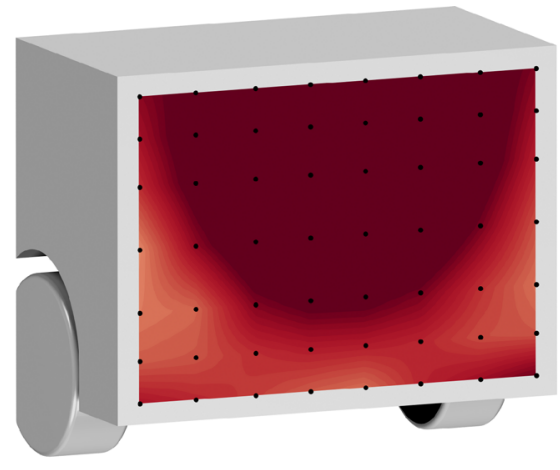

(a) Square-back

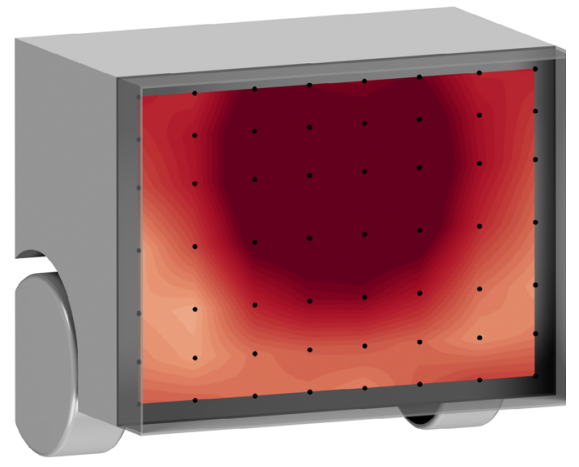

(b) Straight cavity

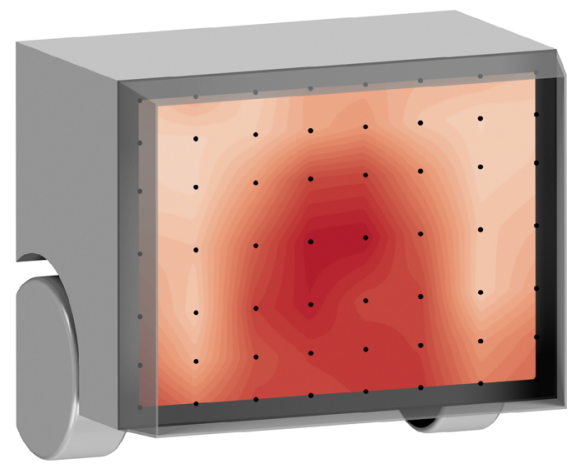

(c) Tapered cavity

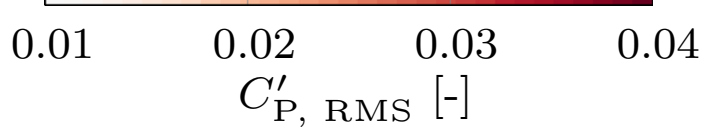

Fig. 13 Base pressure fluctuations at $0^{\circ}$-yaw of the square-back, straight cavity and tapered cavity. The pressure tap locations are marked in black

consistent with the results from Lucas et al. (2017) where different depth cavities were applied to the Ahmed model.

The Power Spectral Density (PSD) of the centre of pressure movement on the base for the lateral and vertical direction is shown in Fig. 15. The large high-frequency peaks

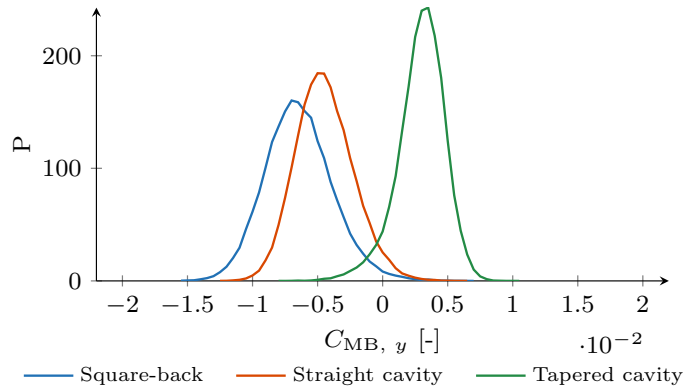

(a) Coefficient of base moment around the horizontal axis at the base midpoint.

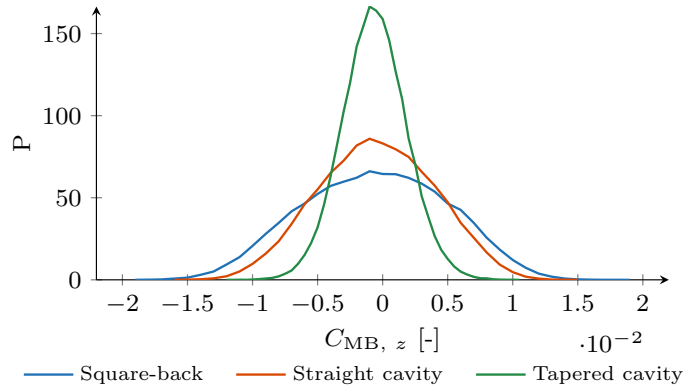

(b) Coefficient of base moment around the vertical axis at the base

Fig. 14 Probability Density Function (PDF) of the base moment coefficient

around $S t=0.7$ are related to the main fan in the wind tunnel and are not related to the flow features at the base (Fuller 2012). The lower-frequency peaks, at $S t=0.08$ and $S t=0.1$, are consistent with the widely reported pumping motion of the wake and the higher peaks, at $S t=0.19$ and $S t=0.23$, to the flapping motion of the wake (Duell and George 1999; Pavia et al. 2020a). Duell and George (1999) describe the pumping motion as a vortex pair leaving the wake causing the bubble pumping of the wake as the vortices are shed. It is expected that the pumping motion has a small effect on the centre of pressure movement as it acts in the streamwise direction primarily. The peaks around $S t=0.08-0.1$ are less prevalent in Fig. 15; however, for both geometries with cavities, there is evidence of the pumping motion in the centre of pressure movement. This suggests that the pumping motion is coupled to the vertical and lateral motion of the wake and not only the longitudinal motion. For the square-back geometry, there is no sign of frequencies associated with the pumping motion in the centre of pressure movement.

Varney (2020) investigated the effects of cavity depth for a straight cavity on the same geometry without wheels. For the square-back configuration, the pumping motion was evident at the base and, as the cavity depth was increased, this wake mode was gradually suppressed and the base drag was reduced. This was ascribed to a damping effect as the wake volume increased with increasing cavity depth, 


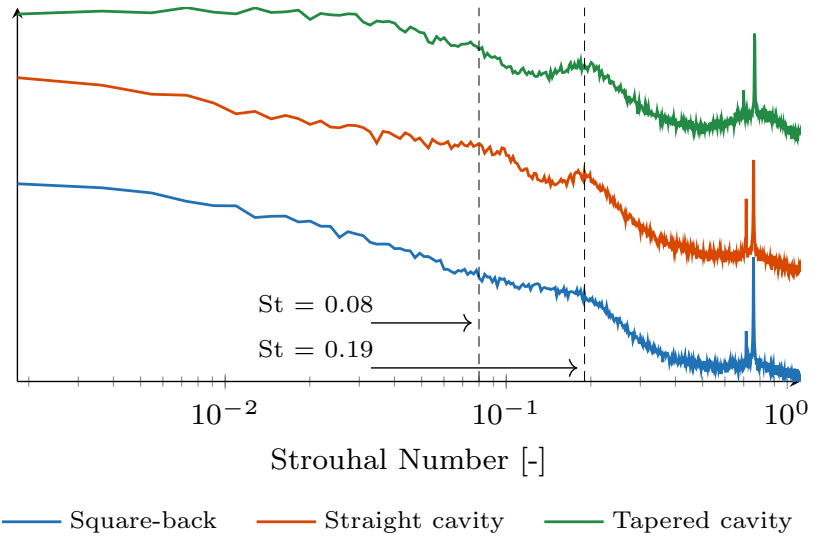

(a) Lateral movement

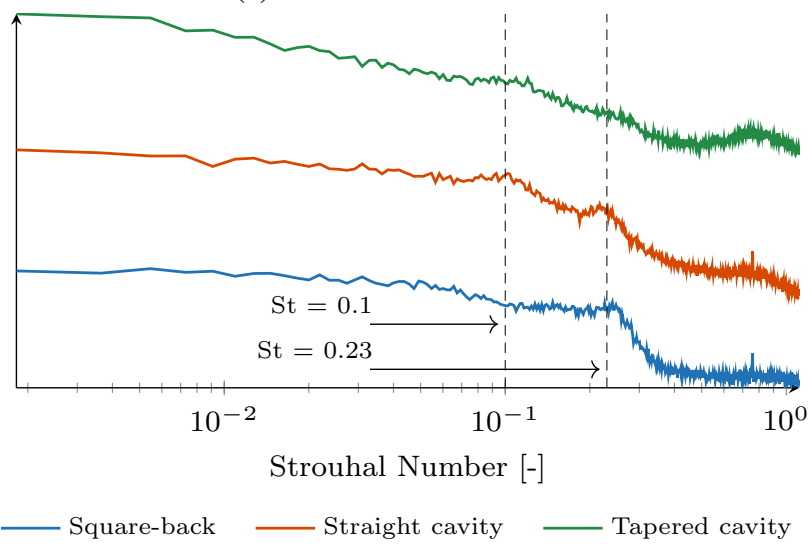

(b) Vertical movement

Fig. 15 Power Spectral Density (PSD) of centre of pressure movement. The Strouhal number is normalised by the square root of the vehicle frontal area and freestream velocity

and the freestream supplying energy to the shear layers is largely unaffected by the cavity depth. The wheels introduce additional upwash compared to the model without wheels. The absence of the pumping frequencies for the squareback geometry could be linked to the upwash, locking the wake into a stabilised state. This stable, but high drag, state hinders the pumping motion of the wake, which is seen on geometries with more balanced wakes (Evrard et al. 2016; Varney 2020). This theory is in line with the results by Bonnavion and Cadot (2018) where a dominant static symmetry breaking mode causes the flow in the perpendicular axis to revert to the mean flow. Here, the addition of the straight cavity causes the wake to become less upwash dominated and thus more balanced. The results indicate that as the wake becomes more balanced it is able to move more freely, reintroducing the pumping mode. Similar results were also found by Haffner et al. (2021) where the vertical symmetrisation of the wake on an Ahmed introduced lateral bi-stability.

The PDF of the centre of pressure movement was also investigated, as shown in Fig. 16. This is different from the

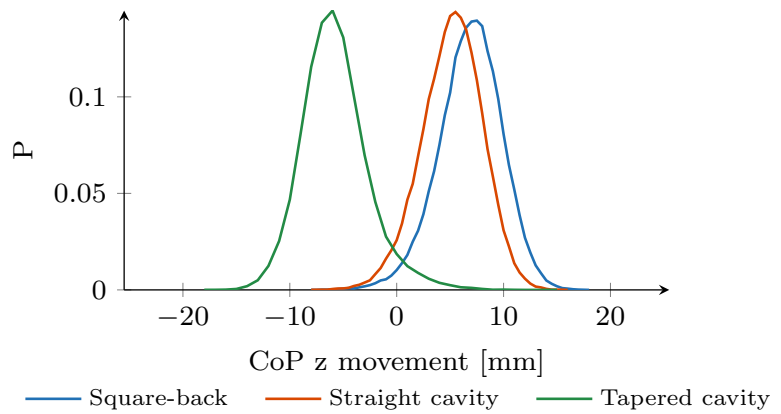

(a) Centre of pressure movement in the vertical direction.

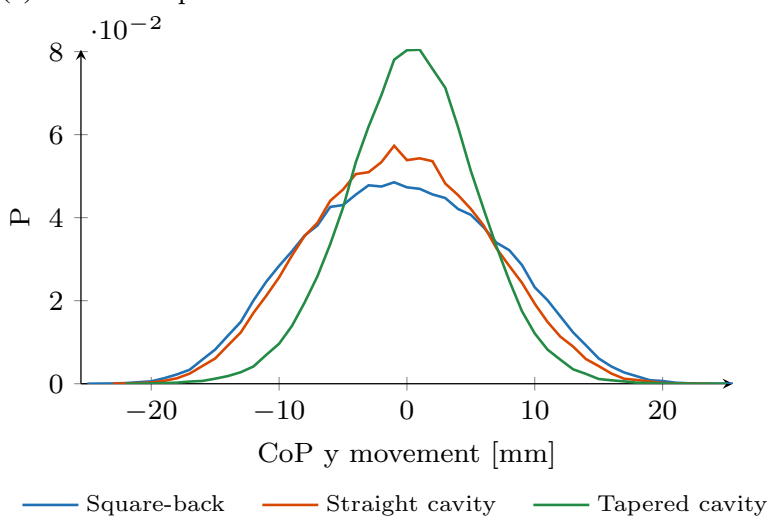

(b) Centre of pressure movement in the horizontal direction.

Fig. 16 Probability Density Function (PDF) of the base centre of pressure. The midpoint of the base is defined as 0

base moment coefficient as it relates to how the pressure balance on the wake is changing while the base moment coefficient relates to the strength of the movement. The distributions are similar in the vertical movement for all three configurations although centred around different base heights. The lateral CoP movement, as shown in Fig. 16b, follows a similar distribution as the lateral moment coefficient, as shown in Fig. 14b, although the differences in the distribution heights are much smaller between each configuration. The smaller differences in CoP distributions in combination with the differences in the base moment coefficient distributions strengthen the theory that the wake is able to move more freely for the more balanced, lower drag, configurations. This can be thought of as a seesaw effect where a lighter seesaw, or reduction in base suction, requires smaller perturbations for the same $(\mathrm{CoP})$ movement.

For further drag reduction, it is beneficial to damp out the large-scale wake motions, for example, by increasing the cavity depth (Varney 2020; Evrard et al. 2016), introducing a control cylinder (Grandemange et al. 2014; Evrard et al. 2016), actively symmetrising the wake with jets ( $\mathrm{Li}$ et al. 2016) or adding a splitter plate (Bearman 1965) forcing the wake to spend more time closer to a balanced state. This can be observed for the tapered cavity where the pressure 
fluctuations are lower and the overall CoP movement is smaller, as shown in Figs. 13 and 16, respectively.

The two-point correlation was investigated for each point in the base. Figure 17 shows the correlation for a tap located near the base central position. Both geometries with cavities have smaller negatively correlated areas in the lateral directions consistent with the description of the pumping motion (Duell and George 1999; Pavia et al. 2020a). The correlation for points located at the sides of the model was also investigated, indicating strong flapping motion for the

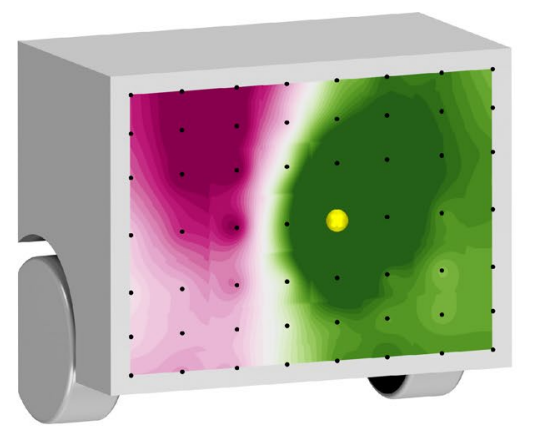

(a) Square-back

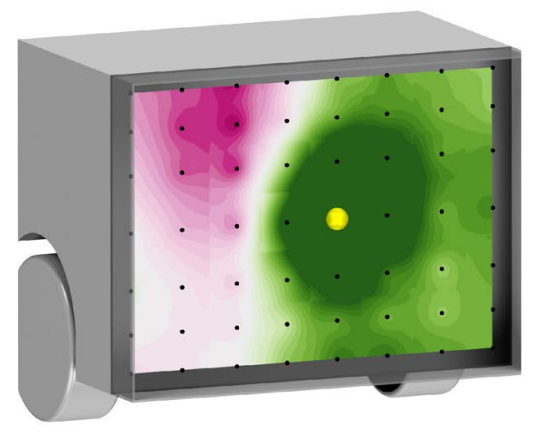

(b) Straight cavity

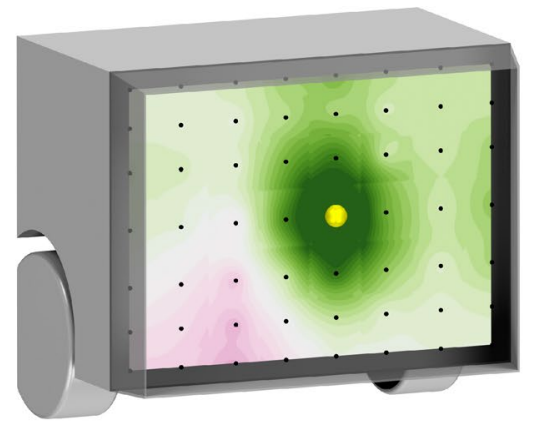

(c) Tapered cavity

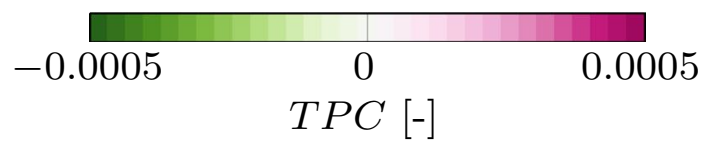

Fig. 17 Two-point correlation at the pressure tap marked with a yellow sphere square-back and straight cavity, not shown here. The top and bottom tap positions were also investigated, but no strong correlation, or flapping, in the vertical direction was found. However, this does not exclude the presence of vertical flapping in the wake; rather, it indicates that the coherent base pressure fluctuations are mainly dominated by the side to side flapping. More energetic lateral flapping is expected in a geometry that has a larger width compared to the height. Generally, the two-point correlation magnitude is smaller for the straight cavity and tapered cavity; this is to be expected as it is related to the base pressure fluctuations. The results indicate that the additional drag reduction from tapering the cavity is primarily due to the reduction in wake size and crossflow. However, the reduction in unsteadiness, allowing the wake to spend more time in a symmetric state, is also related to reductions in drag (Varney 2020).

The crossflow and longitudinal velocities within the wake are defined as

$\overline{C_{\text {crossflow }}} A=\int_{S_{\text {wake }}}\left[\frac{\sqrt{{\overline{v_{y}}}^{2}+{\overline{v_{z}}}^{2}}}{\overline{v_{\infty}}}\right] \mathrm{d} S_{\text {wake }}$

and

$\overline{C_{\text {longitudinal }}} A=\int_{S_{\text {wake }}}\left[\frac{\overline{v_{x}}}{\overline{v_{\infty}}}\right] \mathrm{d} S_{\text {wake }}$,

respectively. The wake surface, $S_{\text {wake }}$, is defined as the region where the longitudinal velocity, $\overline{v_{x}}$, is smaller than $0.25 v_{\infty}$. These, and similar, quantities have been shown to correlate with vehicle drag in previous work (Luckhurst et al. 2019; Urquhart et al. 2020b).

The integrated crossflow is not directly related to drag; instead, it is used here to investigate the previously presented qualitative results and is shown in Fig. 18. Comparing the square-back to the straight cavity, it is observed that the cavity reduces the crossflow in the wake and increases the longitudinal backflow close to the base. The reduction in crossflow in the wake indicates reduced wake losses and has been shown to correlate with a reduction in drag (Urquhart et al. 2018, 2020b).

Comparing the crossflow for the tapered cavity to the filled tapered cavity, the same trend as for the square-back and straight cavity comparison is observed. It is important to note that the trends between all configurations are not as apparent and it is more suitable to use crossflow analysis when the changes are local. For local geometry changes, the crossflow within the wake can be used as a tool to qualitatively compare wakes and find potential improvement areas. For example, areas in the wake with large in- or out-wash can be reduced by angling the trailing edge of the geometry to counteract this. 


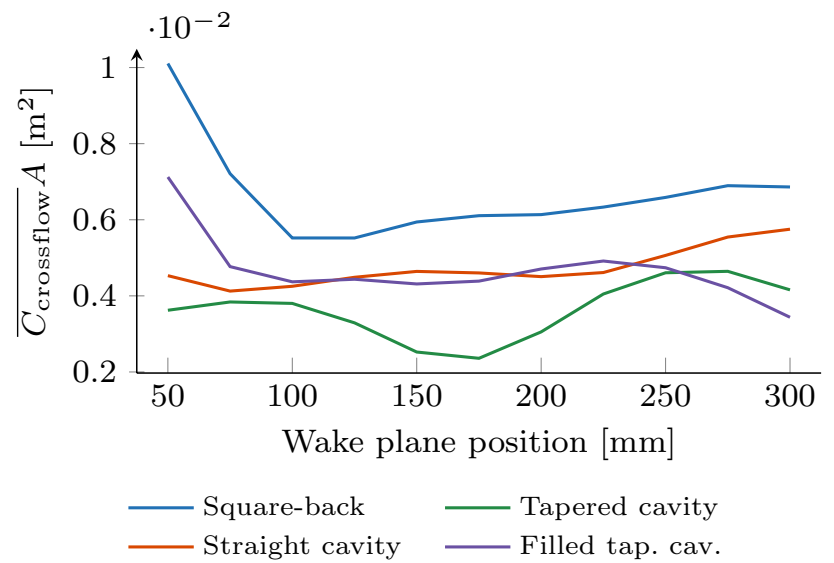

(a) Crossflow

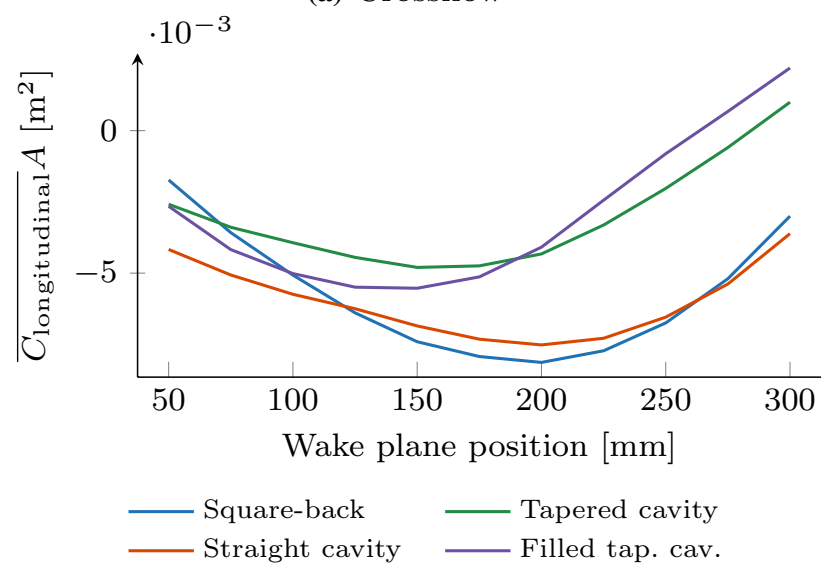

(b) Longitudinal

Fig. 18 Integrated crossflow and longitudinal velocities within the wake. Wake plane distance is referenced relative to the base of the vehicle body (cavity not included). The integration planes used are placed every $25 \mathrm{~mm}$

The larger negative velocity close to the base can be a sign of a wake with lower drag as the kinetic energy of the flow moving towards the base can be converted into an increase in base pressure. However, the longitudinal velocity in the wake is not as consistent at predicting if the drag decreases or increases and has a larger dependence on the downstream location. This might be explained by the lack of directional information when considering the integrated velocity only. Haffner et al. (2021) showed that the shear layer interactions between opposite sides are an important indicator of drag.

\section{$3.25^{\circ} \& 10^{\circ}$-yaw}

The force and base drag values are shown in Table 2 .

Similar to the $0^{\circ}$-yaw condition, the relative order of drag reduction between different geometries is the same. Adding a taper reduces drag with further reductions when combined
Table 2 Aerodynamic force coefficients at yaw

\begin{tabular}{llllll}
\hline Configuration & $C_{\mathrm{D}}$ & $C_{\mathrm{FL}}$ & $C_{\mathrm{RL}}$ & $C_{\mathrm{DB}}$ & $C_{\mathrm{Yaw}}$ \\
\hline $\mathbf{5}^{\circ}$-yaw & & & & & \\
Square-back & 0.435 & 0.171 & 0.008 & 0.322 & -0.082 \\
Straight cavity & 0.425 & 0.174 & -0.003 & 0.308 & -0.081 \\
Tapered cavity & 0.354 & 0.151 & 0.183 & 0.226 & -0.124 \\
Filled tap. cav. & 0.367 & 0.159 & 0.170 & - & -0.119 \\
$\mathbf{1 0}^{\circ}$-yaw & & & & & \\
Square-back & 0.482 & 0.252 & 0.032 & 0.357 & -0.160 \\
Straight cavity & 0.476 & 0.259 & 0.024 & 0.350 & -0.157 \\
Tapered cavity & 0.378 & 0.269 & 0.242 & 0.256 & -0.209 \\
Filled tap. cav. & 0.389 & 0.277 & 0.227 & - & -0.202 \\
\hline
\end{tabular}

with a cavity. Although tapering the cavity reduces drag, it increases lift and yaw moment; both have been identified as negative indicators of high-speed stability by Brandt et al. (2020); the improvements in drag need to be balanced with stability constraints.

The wake along the centreline is upwash dominated for all configurations at $5^{\circ}$-yaw; Fig. 19 shows the tapered cavity wake. Without yaw, the optimised tapered cavity was downwash dominated, as shown in Fig. 10c. This was an unexpected result. Typically, a balanced wake presents a flow that is more perpendicular to the base and is linked to improvements in vehicle drag (Urquhart et al. 2018; Wang et al. 2020; Perry 2016).

The base pressures, as shown in Fig. 20, indicate the typical upwash dominated wake with a high-pressure region in the top windward corner and a low-pressure region in the opposite corner. This explains why the optimisation led to a geometry which is downwash dominated at $0^{\circ}$-yaw, pushing the diffuser angle to the lowest allowed

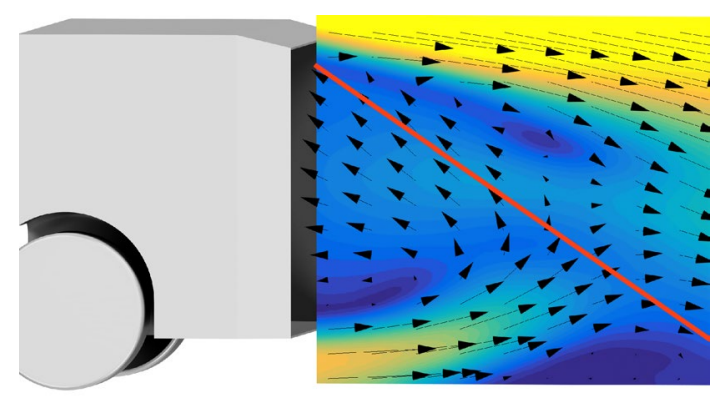

(a) Tapered cavity

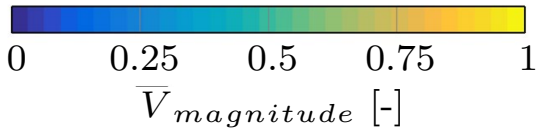

Fig. 19 Time-averaged centreline velocity magnitude at $5^{\circ}$-yaw normalised by the freestream velocity. The line is a qualitative addition to indicate the return flow direction and is placed manually in the region of large backflow velocity 


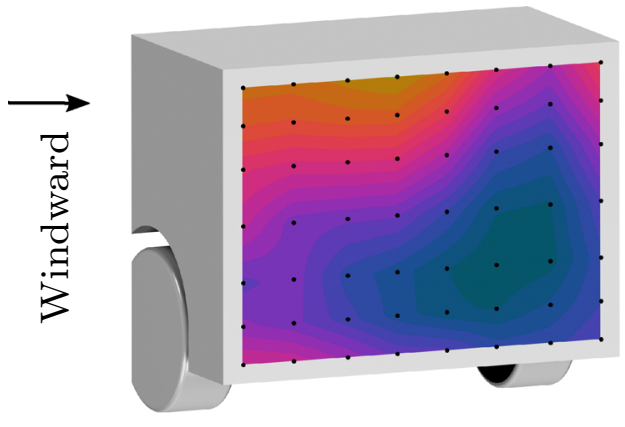

(a) Square-back

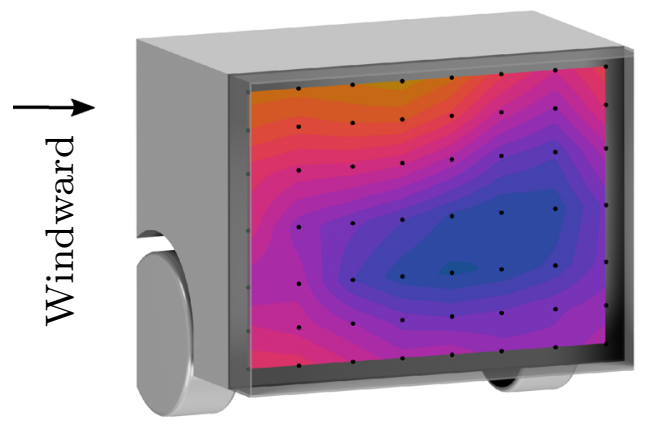

(b) Straight cavity

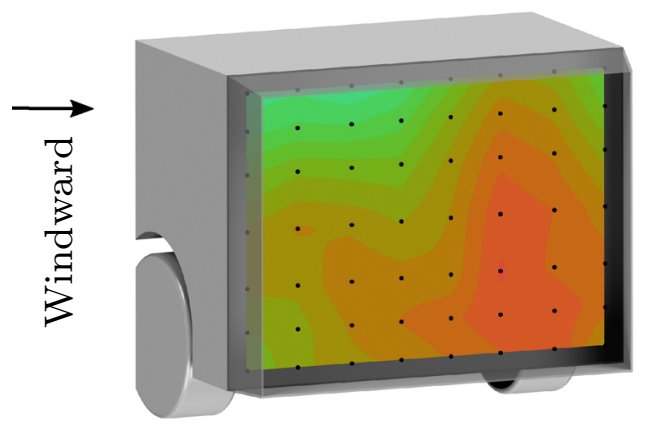

(c) Tapered cavity taper

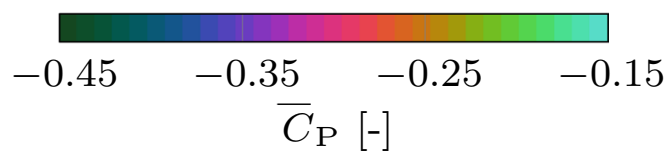

Fig. 20 Time-averaged base pressure at $5^{\circ}$-yaw of the square-back, smooth taper and optimised flap angles. The pressure tap locations are marked in black

angle as the increased asymmetry at yaw causes engulfment of high energy flow into the wake, increasing drag (Haffner et al. 2021). Other designs with lower drag at $0^{\circ}$-yaw were found during the numerical optimisation. These designs often featured a smaller roof taper angle and a larger diffuser angle compared to the design with the best cycle averaged drag performance. The tapered cavity increases the base pressure overall but also reduces the lateral and vertical pressure gradients indicative of a more balanced wake.

Howell (2015) showed that, depending on the vehicle shape, the drag is either positively or negatively correlated with the lift change at yaw. His findings are consistent with different vehicle shapes producing either upwash or downwash dominated wakes. The geometry investigated here is upwash dominated at yaw. A wake that becomes more upwash dominated as the model is yawed benefits from increasing the downwash past the optimum at $0^{\circ}$-yaw to reduce the cycle averaged drag.

As the wake is not symmetric at yaw, it is not appropriate to compare highly 3-dimensional wakes in only one longitudinal plane, and therefore, Fig. 21 shows the crossflow in the near wake behind the vehicle. The upper windward portion of the wake is upwash dominated for all configurations. The crossflow is lower for the straight cavity compared to the square-back, and it is the lowest for the tapered cavity. Filling in the tapered cavity also increases the near wake crossflow velocities.

The integrated crossflow is shown in Fig. 22 where the differences are more apparent. The relative order of different geometries drag values and the near base crossflow is in agreement in this case, which is consistent with other work where low near-wall velocities were linked to decreases in drag (Luckhurst et al. 2019; Varney 2020).

Garcia de la Cruz et al. (2017) and Varney et al. (2018a) have shown that asymmetric side tapering or rearward facing flaps can reduce the drag at yaw. Varney et al. (2018a) attributed the drag reduction to a more symmetric wake as the side force fluctuations, indicative of a balanced wake, increased. Both studies featured positive angles on the leeward side and negative angles on the windward side for the low drag configurations. In previous work, a similar result was found at yaw for the same tapered geometry investigated here with the addition of small flaps that were added at the trailing edge of the cavity (Urquhart et al. 2020b). These flaps reduced the crossflow in the wake and increased the unsteadiness of the wake, creating a more balanced wake with lower drag.

Figure 23 shows the base pressure fluctuations for the three configurations where measurements were taken. Overall, the fluctuations decreased at yaw compared to zero yaw as shown in Fig. 13; however, the relative fluctuations remain the same with the square-back having the highest levels and the tapered cavity having the lowest levels. The turbulent kinetic energy in the wake showed similar trends to the base pressure fluctuations.

The PDF of the base moment coefficient is shown in Fig. 24. The differences between the geometries are less pronounced compared to the $0^{\circ}$-yaw condition, although the tapered cavity has the peaks located closer to the centre for both the lateral and vertical movements, indicating 


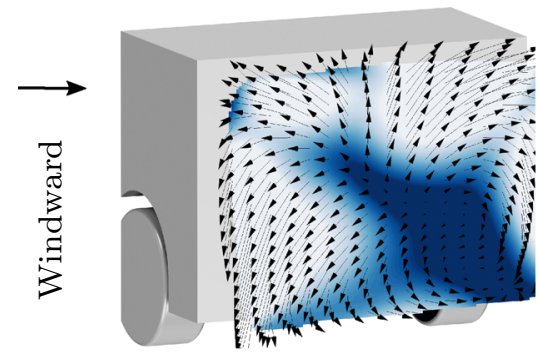

(a) Square-back

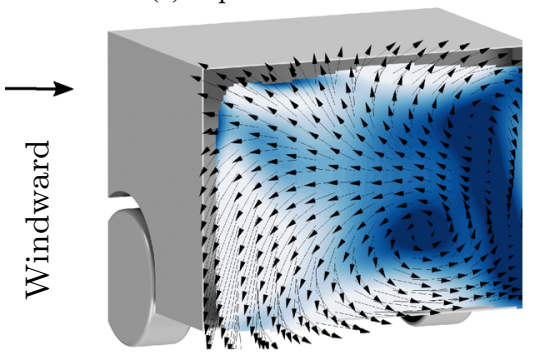

(b) Straight cavity

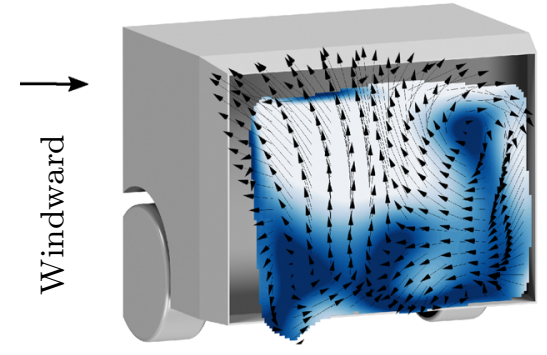

(c) Tapered cavity

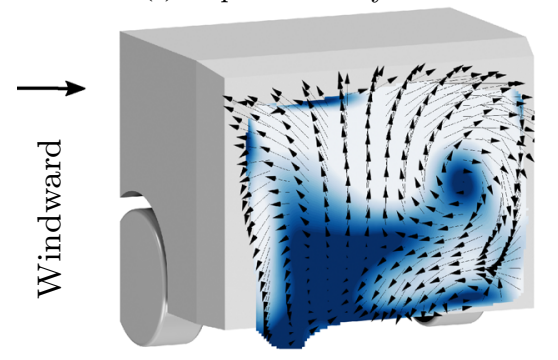

(d) Filled tapered cavity

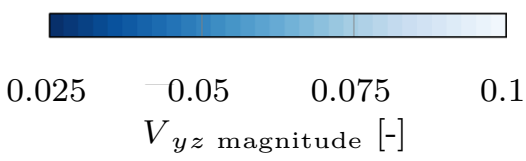

Fig. 21 Crossflow velocity magnitude $75 \mathrm{~mm}$ behind the base of the square-back vehicle. The values are time-averaged and normalised by the freestream velocity. The plane is clipped where the longitudinal velocity is larger than $25 \%$ of the freestream velocity

a more symmetric wake. The square-back has, as expected, the least balanced wake with the largest fluctuations in the base moment coefficient.

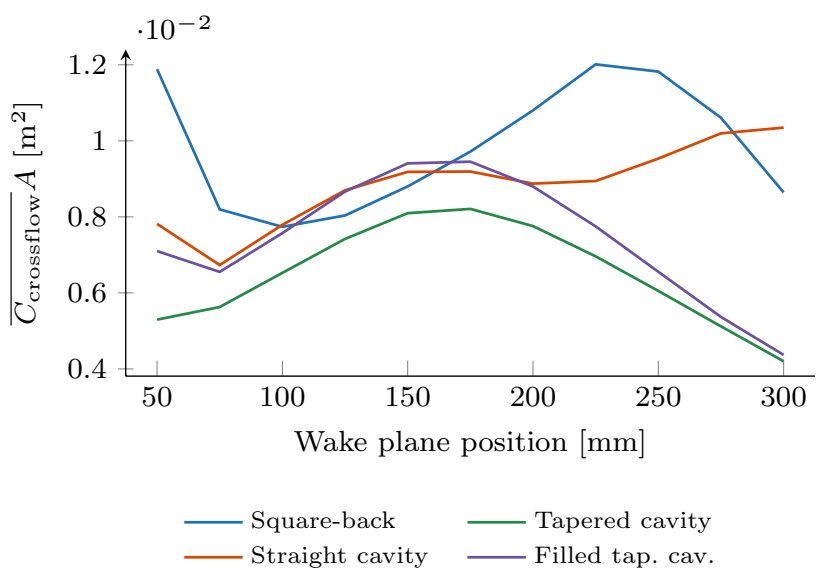

Fig. 22 Integrated crossflow velocities within the wake. Wake plane distance is referenced relative to the base of the vehicle body (cavity not included). The integration planes used are placed every $25 \mathrm{~mm}$

Figure 25 shows the PSD of the CoP movement at $5^{\circ}$ -yaw. In the vertical direction the peaks reduced compared to the $0^{\circ}$-yaw condition, it is hypothesised that this is due to the wakes being locked in an upwash dominated state where the flapping and pumping motions are suppressed. For the lateral movement, the peaks are still retained. It is only the tapered cavity that indicates a clear peak around the pumping frequencies which is thought to be related to the more balanced wake. This also indicates that the tapered cavity could benefit from reducing this motion by, for example, increasing the cavity depth. Larger cavity depths are known to improve the drag at yaw for the straight cavity (Varney 2020).

It is worth noting that the frequency of the peaks has generally been reduced in frequency compared to the $0^{\circ}$ yaw case. It is not clear why the peaks of these frequencies have changed. It could be related to the change in wake size and shear layer interactions. A different scaling of the Strouhal number, for instance, using the wake length or cube root of the wake volume for each configuration, could result in frequency peaks more consistent over different operating conditions.

The two-point correlation was also investigated for the geometries at yaw. Generally, the correlations were more similar in magnitude between the three configurations which is consistent with the magnitude in base pressure fluctuations at yaw. The large in- and out-of-phase correlated areas found at $0^{\circ}$ for the square-back and straight cavity are less evident at yaw which is again expected due to the reduction in the base pressure fluctuations and the less pronounced peak in the spectral content of the CoP movement, as shown in Fig. 25. However, at lower correlation levels, indications of these movements are still present. The two-point correlation results strengthen the theory that wakes become more locked when in the upwash 


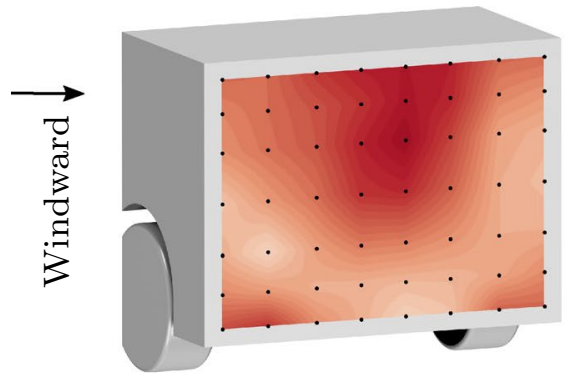

(a) Square-back

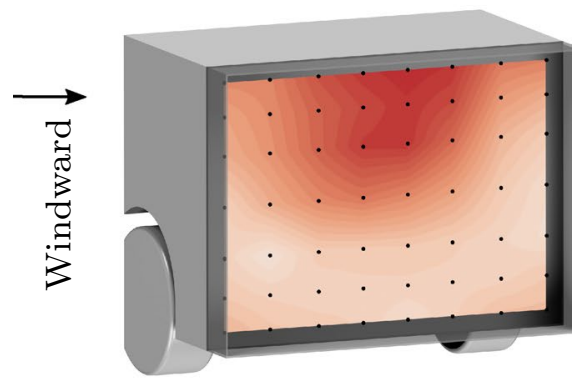

(b) Straight cavity

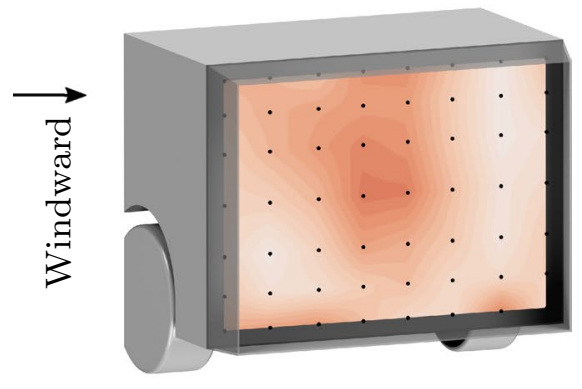

(c) Tapered cavity

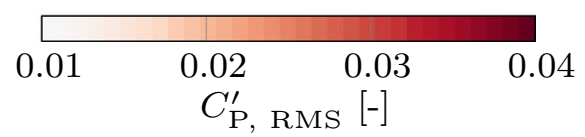

Fig. 23 Base pressure fluctuations at $5^{\circ}$-yaw of the square-back, straight cavity and tapered cavity. The pressure tap locations are marked in black

dominated state at yaw, suppressing the larger flapping and pumping wake motions.

Figure 26 shows the vorticity in the shear layers at the centre of the base for the square-back and straight cavity. With the cavity, the time-averaged vortex at the leeward side of the wake moves towards the base. This influences the flow near the trailing edge where the flow in the cavity is more aligned to the freestream at the cavity edge. This is similar for the windward side resulting in more concentrated shear layers with larger velocity gradients, indicative of less shear layer mixing. The reduction in mixing across the shear layers can be a result of the more aligned flow and lower velocity

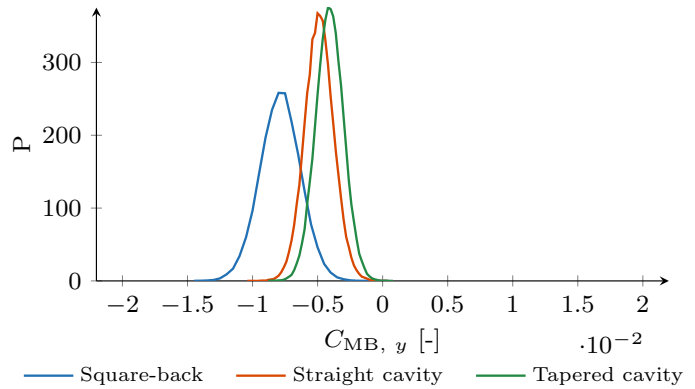

(a) Coefficient of base moment around the horizontal axis at the base mid-point.

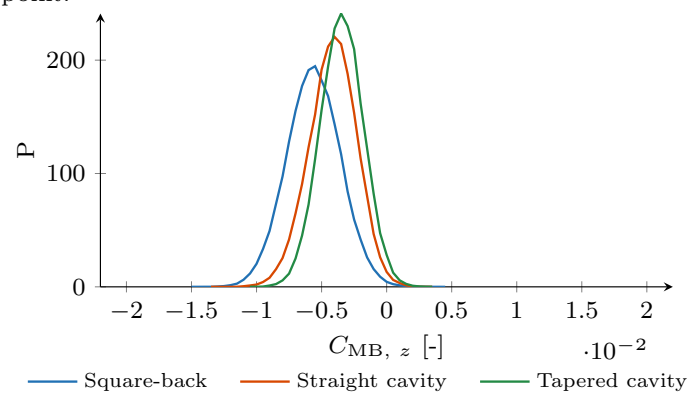

(b) Coefficient of base moment around the vertical axis at the base mid-point.

Fig. 24 Probability Density Function (PDF) of the base moment coefficient

gradient at the trailing edge of the cavity, reducing drag. The internal walls of the cavity also provide resistance to the crossflows as they provide solid boundaries where kinetic energy in the wake can be converted to pressure. This creates a negative feedback loop, re-aligning the instantaneous recirculating flow towards the mean. This contributes to the damping effect of cavities in conjunction with the added mass in the wake as discussed by Varney (2020). The results are similar for the tapered cavity and the filled tapered cavity, although the differences in the vorticity concentration in the shear layer are less pronounced.

A complete view of the highest and lowest drag wake is shown in Fig. 27a and 27b, respectively, where the more concentrated vorticity in the wake shear layer can be seen for the low drag, tapered cavity. The tapered cavity wake is smaller in width and length compared to the square-back geometry.

Figure 28 shows the drag for each yaw angle as well as the driving cycle equivalent drag value, Equation (1).

In total, a $18.6+-0.3 \%$ reduction in the cycle averaged drag is achieved using the tapered cavity over the squareback. The straight cavity improves the cycle averaged drag by approximately $4.7+-0.3 \%$ compared to the square-back and $3.9+-0.4 \%$ for the tapered cavity compared to the filled tapered cavity geometry. The results at yaw show that it is primarily a change to the mean flow which reduces the drag. This also indicates that the potential for improvements in 


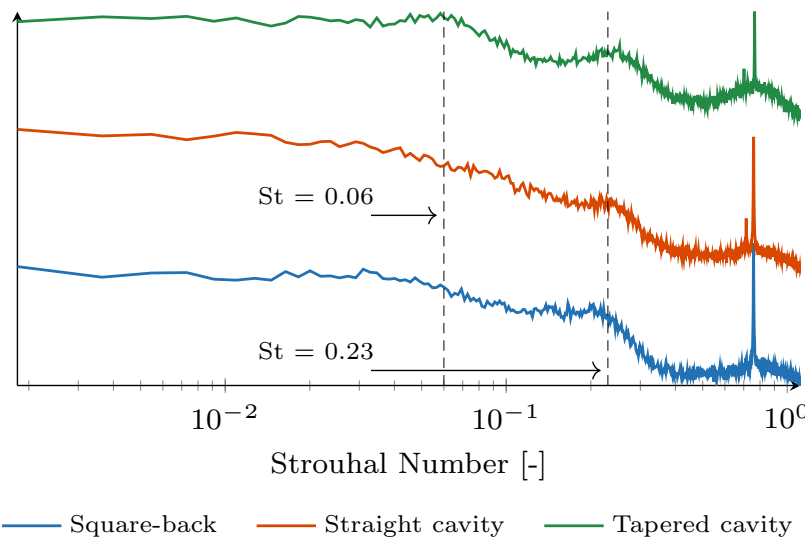

(a) Lateral movement

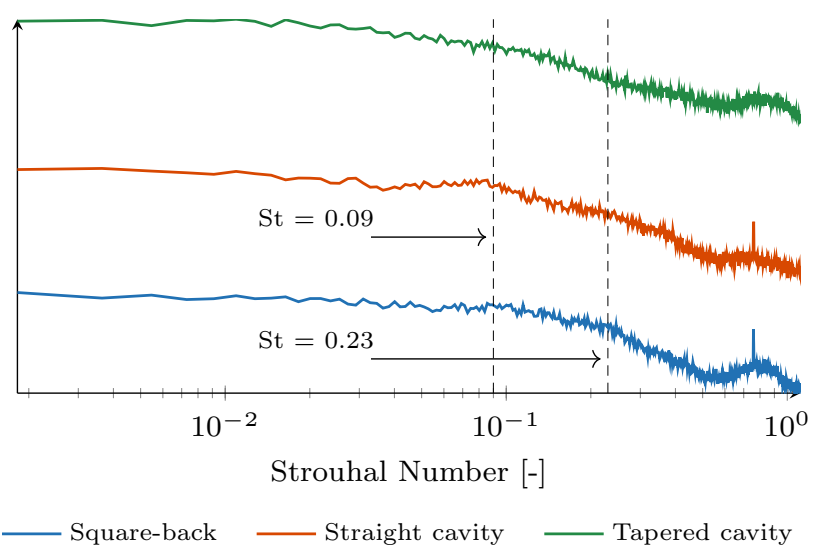

(b) Vertical movement

Fig. 25 Power Spectral Density (PSD) of centre of pressure movement. The Strouhal number is normalised by the square root of the vehicle frontal area and freestream velocity

drag from improving the unsteady dynamics of the wake at yaw is limited compared to $0^{\circ}$-yaw.

The optimisation with RANS simulations used to create the geometry predicted a $14.7 \%$ improvement from the tapered cavity over the square-back. Although the simulated predictions differ from the wind tunnel results, the use of RANS simulations for optimisation highlights the usefulness of lower fidelity, lower cost, but fast simulations to optimise the vehicle geometry. There are also several documented improvements that can be made to the optimisation process, for example, Nagawkar et al. (2020) combined results from low fidelity simulations and high fidelity simulations to create an affordable, but more accurate, surrogate model.

\section{Concluding remarks}

This work focused on a straight and tapered base cavity on a simplified vehicle which were tested in a model scale wind tunnel. Prior to the experimental study, the angles of

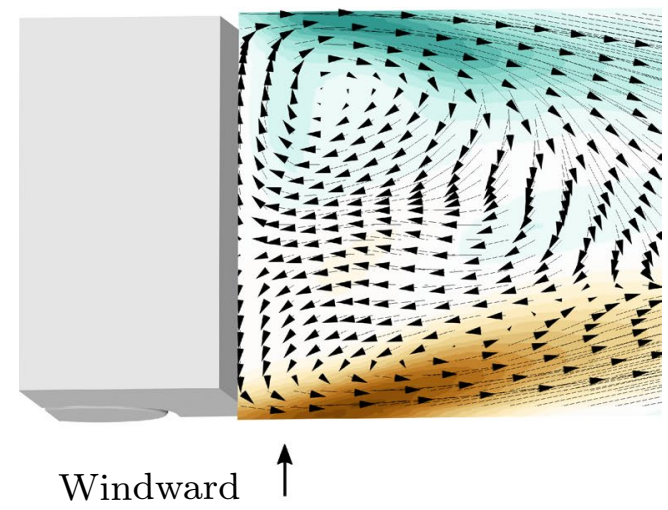

(a) Square-back

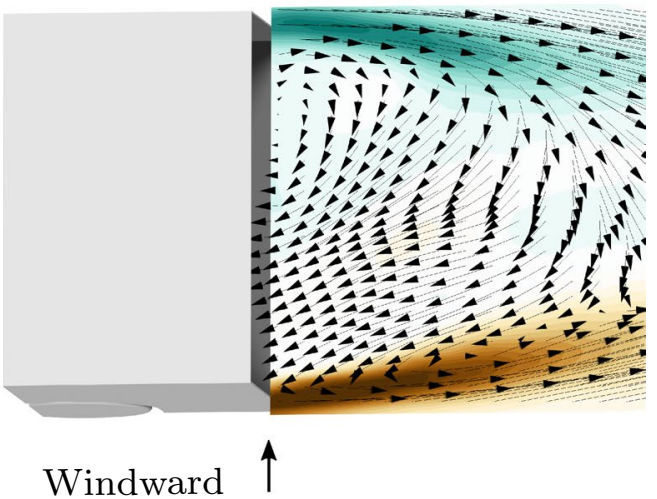

(b) Straight cavity

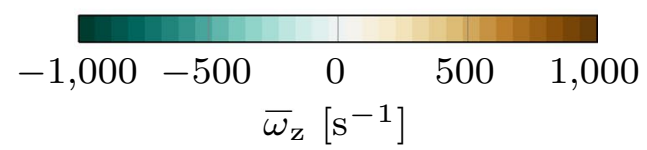

Fig. 26 Time-averaged vorticity along the lift axis at nicefrac 12 of the base height at $5^{\circ}$-yaw with vectors of time-averaged velocity

the cavity were optimised to minimise cycle averaged drag using low-cost steady-state numerical simulations and a surrogate model based optimisation algorithm. The simulated improvement of $15 \%$ was surpassed in the wind tunnel tests where a reduction of approximately $19 \%$ in the cycle averaged drag was achieved using the optimised tapered cavity. The optimised geometry has a more balanced wake at different yaw conditions by inducing downwash with the top and bottom taper angles. This downwash incurs a small penalty in drag at $0^{\circ}$-yaw due to the vertical wake asymmetry but increases the robustness over the operating range.

There are several mechanisms in the wake contributing the drag reduction. Overall unsteadiness in the wake is reduced by adding the straight cavity, which is further reduced with tapering. Adding a straight cavity provides similar improvements in base drag and overall drag. However, the base drag improvement for a tapered cavity is significantly higher than the overall drag improvement 


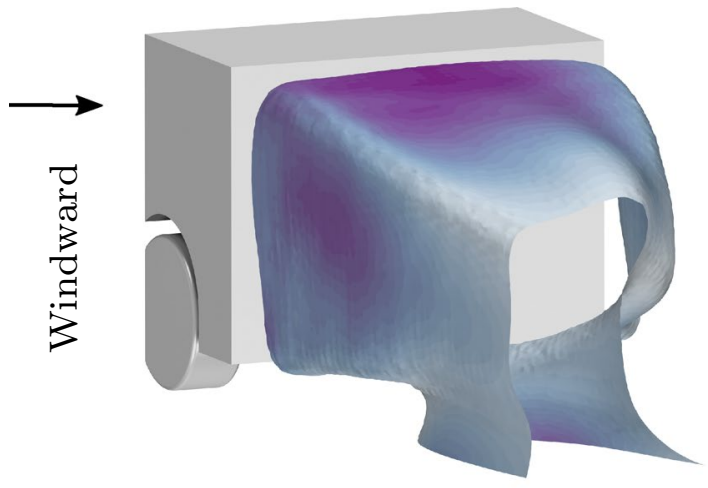

(a) Square-back

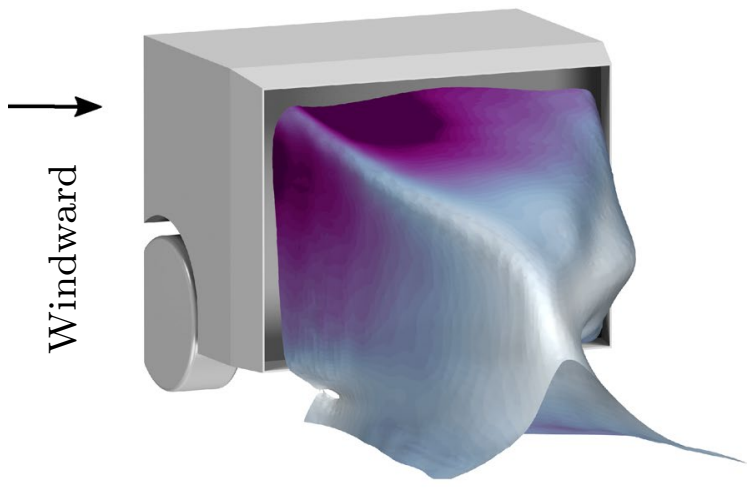

(b) Tapered cavity

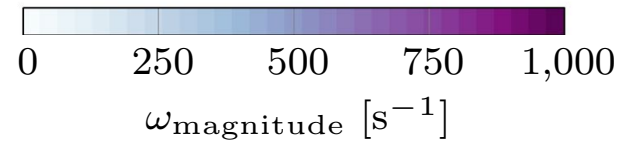

Fig. 27 Time-averaged ISO surface of $\bar{V}_{x}$ at $7.5 \mathrm{~m} / \mathrm{s}$ for $5^{\circ}$-yaw coloured by the time-averaged vorticity magnitude

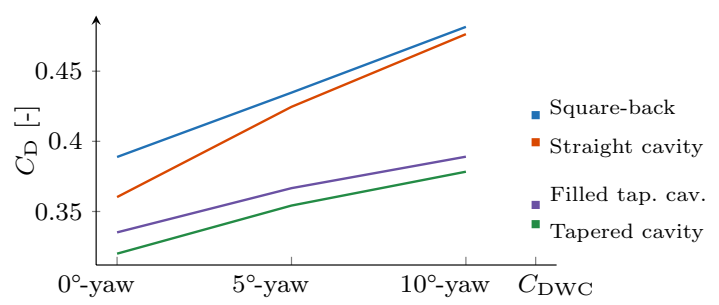

Fig. 28 Drag for each yaw angle and the driving cycle equivalent drag value

due to the drag penalty on the outer tapered surfaces which should be considered during design. The large scale motions associated with wake flapping and pumping become more evident as the balance of the wake is improved when adding the cavities. An unbalanced wake that is either upwash or downwash dominated shows suppression of these modes. However, the results indicate that improving the wake balance decreases overall drag even though the large-scale coherent wake motion increases, which is supported by the literature.

Tomographic PIV demonstrates that the flow in the recirculating wake meets the freestream flow in the shear layers with a smaller difference in angle when the cavity is used. The turbulent kinetic energy in the shear layers decreases with the cavities, with less entrainment in the wake which reduces the shear layer thickness. The added mass of the wake that results from applying the cavity, in combination with the improved shear layer flow, as well as the internal walls of the cavity, all contributes to the decrease in wake unsteadiness.

The near wake crossflow is reduced when adding a cavity and by optimising the taper angle of the cavity, this is further decreased. This is in agreement with previous work where lower levels of near wake crossflow are linked to improvements in drag. In this work, crossflow is found to be one of the more important qualitative indicators of overall drag.

Acknowledgements The authors thank Matthew Ward for all the help and assistance during the wind tunnel tests. The authors would also like to thank Alexander Broniewicz and Lennert Sterken from the Volvo Car Corporation for their valuable input and support throughout this work. The simulations were performed on resources provided by the Swedish National Infrastructure for Computing (SNIC) at NSC. (SNIC 2019)

Funding Open access funding provided by Chalmers University of Technology. This work is funded by the Swedish Energy Agency grant number P43328-1.

\section{Declarations}

Conflict of interest The authors declare that they have no conflict of interest.

Open Access This article is licensed under a Creative Commons Attribution 4.0 International License, which permits use, sharing, adaptation, distribution and reproduction in any medium or format, as long as you give appropriate credit to the original author(s) and the source, provide a link to the Creative Commons licence, and indicate if changes were made. The images or other third party material in this article are included in the article's Creative Commons licence, unless indicated otherwise in a credit line to the material. If material is not included in the article's Creative Commons licence and your intended use is not permitted by statutory regulation or exceeds the permitted use, you will need to obtain permission directly from the copyright holder. To view a copy of this licence, visit http://creativecommons.org/licenses/by/4.0/.

\section{References}

Adrian R, Yao CS (1985) Pulsed laser technique application to liquid and gaseous flows and the scattering power of seed materials. Appl Opt 24(1):44-52 (ISSN 0003-6935)

Ahmed SR, Ramm G, Faltin G (1984) Some salient features of the time-averaged ground vehicle wake. SAE International Congress 
and Exposition, ISSN. https://doi.org/10.4271/840300 (ISSN 0148-7191)

Atkinson C, Soria J (2009) An efficient simultaneous reconstruction technique for tomographic particle image velocimetry. Exp Fluids 47(4-5):553. https://doi.org/10.1007/s00348-009-0728-0

Barros D, Borée J, Noack BR, Spohn A, Ruiz T (2016) Bluff body drag manipulation using pulsed jets and Coanda effect. J Fluid Mech 805:422-459. https://doi.org/10.1017/jfm.2016.508 (ISSN 0022-1120, 1469-7645.)

Bates S, Sienz J, Toropov V (2004) Formulation of the optimal latin hypercube design of experiments using a permutation genetic algorithm. Struct Dyn Mater Conf 2011:04. https://doi.org/10. 2514/6.2004-2011

Bearman PW (1965) Investigation of the flow behind a two-dimensional model with a blunt trailing edge and fitted with splitter plates. J Fluid Mech 21(02):241. https://doi.org/10.1017/S0022 112065000162 (ISSN 0022-1120, 1469-7645)

Bonitz S (2018) Development of Separation Phenomena on a Passenger Car. Chalmers University of Technology, ISBN 978-91-7597785-0. URL https://research.chalmers.se/en/publication/510047

Bonnavion G, Cadot O (2018) Unstable wake dynamics of rectangular flat-backed bluff bodies with inclination and ground proximity. $\mathrm{J}$ Fluid Mech 854:196-232

Bonnavion G, Cadot O, Herbert V, Parpais S, Vigneron R, Délery J (2019) Asymmetry and global instability of real minivans' wake. J Wind Eng Ind Aerodyn 184:77-89. https://doi.org/10.1016/j. jweia.2018.11.006

Brandt A, Sebben S, Jacobson B, Preihs E, Johansson I (2020) Quantitative high speed stability assessment of a sports utility vehicle and classification of wind gust profiles. In: WCX SAE World Congress Experience, pages 2020-01-0677. SAE International, April 2020. https://doi.org/10.4271/2020-01-0677

Caridi GCA, Ragni D, Sciacchitano A, Scarano F (2016) HFSB-seeding for large-scale tomographic PIV in wind tunnels. Exp Fluids 57(12):1-13. https://doi.org/10.1007/s00348-016-2277-7 (ISSN 07234864)

Cooper KR(1985) The effect of front-edge rounding and rear-edge shaping on the aerodynamic drag of bluff vehicles in ground proximity. In: SAE international congress and exposition. SAE International, feb . https://doi.org/10.4271/850288

Duell EG, George AR (1993) Measurements in the unsteady near wakes of ground vehicle bodies. SAE Tech Pap SAE Int. https:// doi.org/10.4271/930298 (( ISBN 4230824815))

Duell EG, George AR (1999) Experimental study of a ground vehicle body unsteady near wake. Int Congr Expo SAE Int. https://doi. org/10.4271/1999-01-0812

Elsinga GE, Scarano F, van Oudheusden Wieneke B (2006) Tomographic particle image velocimetry. Exp. Fluids 41(6):933-947. https://doi.org/10.1007/s00348-006-0212-z (( ISSN 1432-1114.))

Elsinga GE, Westerweel J, Scarano F, Novara M (2011) On the velocity of ghost particles and the bias errors in TomographicPIV. Exp Fluids 50(4):825-838 (ISSN 1432-1114. 10.1007/ s00348-010-0930-0)

Evrard A, Cadot O, Herbert V, Ricot D, Vigneron R, Délery J (2016) Fluid force and symmetry breaking modes of a 3D bluff body with a base cavity. J Fluids Struct 61:99-114. https://doi.org/10.1016/j. jfluidstructs.2015.12.001 (ISSN 08899746.)

Favre T, Efraimsson G (2011) An assessment of detached-eddy simulations of unsteady crosswind aerodynamics of road vehicles. Flow Turbul Combust 87(1):133-163. https://doi.org/10.1007/s10494011-9333-4 (ISSN 13866184)

Fuller J B (2012). The unsteady aerodynamics of static and oscillating simple automotive bodies. thesis, Loughborough University, URL https://repository.lboro.ac.uk/articles/thesis/The_unsteady_aerod ynamics_of_static_and_oscillating_simple_automotive_bodies/ 9217136/1
Garcia de la Cruz JM, Brackston RD, Morrison JF (2017). Adaptive base-flaps under variable cross-wind

Gaylard AP, Oettle N, Gargoloff J, Duncan B (2014) Evaluation of non-uniform upstream flow effects on vehicle aerodynamics. SAE Int J Passenger Cars - Mech Syst 7(2):692-702. https://doi.org/10. 4271/2014-01-0614 (ISSN 1946-4002.)

Grandemange M (2013) Analysis and control of three-dimensional turbulent wakes: from axisymmetric bodies to road vehicles. PhD thesis, ENSTA Paris

Grandemange M, Gohlke M, Cadot O (2013) Bi-stability in the turbulent wake past parallelepiped bodies with various aspect ratios and wall effects. Phys Fluids. https://doi.org/10.1063/1.4820372 (ISSN 1070-6631)

Grandemange M, Gohlke M, Cadot O (2014) Turbulent wake past a three-dimensional blunt body. Part 2. Experimental sensitivity analysis. J Fluid Mech 752:439-461. https://doi.org/10.1017/ jfm.2014.345 (ISSN 0022-1120, 1469-7645)

Haffner Y, Borée J, Spohn A, Castelain T (2020) Unsteady Coanda effect and drag reduction for a turbulent wake. J Fluid Mech. https://doi.org/10.1017/jfm.2020.494 (ISSN 0022-1120)

Haffner Y, Borée J, Spohn A, Castelain T (2020) Mechanics of bluff body drag reduction during transient near-wake reversals. J Fluid Mech. https://doi.org/10.1017/jfm.2020.275 (ISSN 00221120, 1469-7645)

Haffner Y, Castelain T, Borée J, Spohn A (2021) Manipulation of three-dimensional asymmetries of a turbulent wake for drag reduction. J Fluid Mech. https://doi.org/10.1017/jfm.2020.1133 (ISSN 0022-1120, 1469-7645)

Han X, Krajnović S, Basara B (2013) Study of active flow control for a simplified vehicle model using the PANS method. Int $\mathrm{J}$ Heat Fluid Flow. https://doi.org/10.1016/j.ijheatfluidflow.2013. 02.001 (ISSN 0142727X)

Howell J (2015) Aerodynamic drag of passenger cars at yaw. SAE Int J Passenger Cars - Mech Syst 8(1)https://doi.org/10.4271/ 2015-01-1559. (ISSN 1946-4002.)

Howell J, Le Good G (1999) The influence of aerodynamic lift on high speed stability. SAE trans. https://doi.org/10.4271/ 1999-01-0651

Howell J, Passmore M, Tuplin S (2013) Aerodynamic drag reduction on a simple car-like shape with rear upper body taper. SAE Int J Passeng Cars - MechInt J Passeng Cars - Mech Syst 6:52-60. https://doi.org/10.4271/2013-01-0462 (ISSN 1946-4002)

Howell J, Passmore M, Windsor S (2018) A drag coefficient for test cycle application. SAE Int J Passenger Cars - Mech Syst 11(5):447-461. https://doi.org/10.4271/2018-01-0742 (ISSN 1946-4002.)

J1594. Vehicle Aerodynamics Terminology (J1594 Ground Vehicle Standard) - SAE Mobilus. URL https://saemobilus.sae.org/conte $\mathrm{nt} / \mathrm{j} 1594 \_199412$

Johl G (2010) The design and performance of a $1.9 m \times 1.3 m$ indraft wind tunnel. $\mathrm{PhD}$ thesis, Loughborough University,

Josefsson E, Hagvall R, Urquhart M, Sebben S (2018) Numerical analysis of aerodynamic impact on passenger vehicles during cornering

Kühn M, Ehrenfried K, Bosbach J, Wagner C (2011) Large-scale tomographic particle image velocimetry using helium-filled soap bubbles. Exp Fluids 50(4):929-948. https://doi.org/10.1007/s00348010-0947-4 (ISSN 07234864)

Li R, Barros D, Borée D, Cadot O, Noack BR, Cordier L (2016) Feedback control of bimodal wake dynamics. Exp Fluids 57(10): 158. https://doi.org/10.1007/s00348-016-2245-2 (ISSN 0723-4864, 1432-1114)

Li R, Borée J, Noack BR, Cordier L, Harambat F (2019) Drag reduction mechanisms of a car model at moderate yaw by bi-frequency forcing. Phys Rev Fluids 4(3):034604 
Lorite-Díez M, Jiménez-González JI, Pastur L, Martínez-Bazán C, Cadot O (2020) Experimental analysis of the effect of local base blowing on three-dimensional wake modes. J Fluid Mech. https:// doi.org/10.1017/jfm.2019.917 (ISSN 0022-1120, 1469-7645)

Lorite-Díez M, Jiménnez-González JI, Pastur L, Cadot O, MartínezBazán C (2020a) Drag reduction on a three-dimensional blunt body with different rear cavities under cross-wind conditions. J Wind Eng Ind Aerodyn 200:104145. https://doi.org/10.1016/j. jweia.2020.104145

Lucas J-M, Cadot O, Herbert V, Parpais S, Délery J (2017) A numerical investigation of the asymmetric wake mode of a squareback Ahmed body - effect of a base cavity. J Fluid Mech 831:675697. https://doi.org/10.1017/jfm.2017.654 (ISSN 0022-1120, 1469-7645.)

Luckhurst S, Varney M, Xia H, Passmore MA, Gaylard A (2019) Computational investigation into the sensitivity of a simplified vehicle wake to small base geometry changes. J Wind Eng Ind Aerodyn. https://doi.org/10.1016/j.jweia.2018.12.010 (ISSN 0167-6105)

Mariotti A, Buresti G, Gaggini G, Salvetti MV (2017) Separation control and drag reduction for boat-tailed axisymmetric bodies through contoured transverse grooves. J Fluid Mech 832:514-549. https://doi.org/10.1017/jfm.2017.676 (ISSN 0022-1120, 14697645. .Publisher: Cambridge University Press)

Nagawkar J, Leifsson LT, Du X (2020) Applications of polynomial chaos-based cokriging to aerodynamic design optimization benchmark problems. In: AIAA Scitech 2020 Forum. American Institute of Aeronautics and Astronautics, https://doi.org/10.2514/6. 2020-0542

Pavia G, Passmore M, Sardu C (2018) Evolution of the bi-stable wake of a square-back automotive shape. Exp Fluids 59(1):1-20. https:// doi.org/10.1007/s00348-017-2473-0 (ISSN 07234864.)

Pavia G, Varney M, Passmore M, Almond M (2019) Three dimensional structure of the unsteady wake of an axisymmetric body. Phys Fluids 31(2):025113. https://doi.org/10.1063/1.5078379 (ISSN 1070-6631)

Pavia G, Passmore MA, Varney M, Hodgson G (2020a) Salient threedimensional features of the turbulent wake of a simplified squareback vehicle. J Fluid Mech 888:A33. https://doi.org/10.1017/jfm. 2020.71. (ISSN 0022-1120, 1469-7645.)

Pavia G, Passmore MA, Varney M, Hodgson G (2020b) Salient threedimensional features of the turbulent wake of a simplified squareback vehicle. J Fluid Mech 888:A33. https://doi.org/10.1017/jfm. 2020.71

Pavia G, Passmore M (2018). Characterisation of Wake Bi-stability for a Square-Back Geometry with Rotating Wheels. In Jochen Wiedemann, editor, Prog. Veh. Aerodyn. Therm. Manag., Cham, Springer International Publishing. p 93-109 https://doi.org/10. 1007/978-3-319-67822-1_6

Pavlovic J, Marotta A, Ciuffo B (2016) CO 2 emissions and energy demands of vehicles tested under the NEDC and the new WLTP type approval test procedures. Appl Energy 177:661-670 (ISSN 03062619)

Perry AK (2016) An investigation into the base pressure of simplified automotive squareback geometries. URL https://dspace.lboro.ac. uk/2134/22605

Perry AK, Almond M, Passmore M, Littlewood R (2016) The study of a bi-stable wake region of a generic Squareback vehicle using Tomographic PIV. SAE World Congr 5:743 (ISSN 1946-4002)

Pfeiffer J, King R (2018) Robust control of drag and lateral dynamic response for road vehicles exposed to cross-wind gusts. Exp Fluids 59(3):45

Scarano F (2013) Tomographic PIV: principles and practice. Meas Sci Technol 24(1):12001. https://doi.org/10.1088/0957-0233/24/1/ 012001. (ISSN 0957-0233)

Scarano F, Ghaemi S, Caridi GCA, Bosbach J, Dierksheide U, Sciacchitano A (2015) On the use of helium-filled soap bubbles for large-scale tomographic PIV in wind tunnel experiments. Exp Fluids 56(2):42. https://doi.org/10.1007/s00348-015-1909-7 (ISSN 1432-1114)

Schuetz T C(2015) Aerodynamics of Road Vehicles, Fifth Edition. SAE International, Warrendale, Pennsylvania, 5 edition edition, ISBN 978-0-7680-7977-7

Shur ML, Spalart PR, Strelets MKh, Travin AK (2008) A hybrid RANS-LES approach with delayed-DES and wall-modelled LES capabilities. Int J Heat Fluid Flow 29(6):1638-1649 (ISSN 0142-727X.)

SNIC. Swedish national infrastrucutre for computing, 2019. URL http://www.snic.se/

Sterken L, Löfdahl L, Sebben S, Walker T (2014) Effect of rear-end extensions on the aerodynamic forces of an SUV. In SAE Technical Papers

Tunay T, Firat E, Sahin B (2018) Experimental investigation of the flow around a simplified ground vehicle under effects of the steady crosswind. Int J Heat Fluid Flow 71(137-152):6. https://doi.org/ 10.1016/j.ijheatfluidflow.2018.03.020 (ISSN 0142727X.)

Urquhart M, Sebben S, Sterken L (2018) Numerical analysis of a vehicle wake with tapered rear extensions under yaw conditions. J Wind Eng Ind Aerodyn 179:308-318. https://doi.org/10.1016/j. jweia.2018.06.001. (ISSN 0167-6105.)

Urquhart M, Ljungskog E, Sebben E (2020) Surrogate-based optimisation using adaptively scaled radial basis functions. Appl Soft Comput. https://doi.org/10.1016/j.asoc.2019.106050 (ISSN 1568-4946)

Urquhart M, Varney M, Sebben S, Passmore M (2020) Aerodynamic drag improvements on a square-back vehicle at yaw using a tapered cavity and asymmetric flaps. Int J Heat Fluid Flow. https://doi.org/10.1016/j.ijheatfluidflow.2020.108737 (ISSN 0142-727X.)

Varney M (2020) Base drag reduction for squareback road vehicles. thesis, Loughborough University, URL https://repository.lboro. ac.uk/articles/Base_drag_reduction_for_squareback_road_vehic les/11823759

Varney M, Passmore M, Gaylard A (2018a) Parametric Study of Asymmetric Side Tapering in Constant Cross Wind Conditions. In SAE Int J Passeng Cars - Mech Syst 11(3):213-224. https://doi.org/10. 4271/2018-01-0718

Varney M, Passmore M, . Gaylard A (2018b). Parametric study of asymmetric side tapering in constant cross wind conditions, jun 2 ISSN 1946-3995

Volpe R, Devinant P, Kourta A (2015) Experimental characterization of the unsteady natural wake of the full-scale square back Ahmed body: flow bi-stability and spectral analysis. Exp Fluids 56(5):1

Wang S, Avadiar T, Thompson MC, Burton D (2019) Effect of moving ground on the aerodynamics of a generic automotive model: the drivaer-estate. J Wind Eng Ind Aerodyn 195:104000

Wang Y, Sicot C, Borée J, Grandemange M (2020) Experimental study of wheel-vehicle aerodynamic interactions. J Wind Eng Ind Aerodyn. https://doi.org/10.1016/j.jweia.2019.104062 (ISSN 0167-6105)

Windsor S (2014) Real world drag coefficient - is it wind averaged drag? In: the international vehicle aerodynamics conference, pages 3-17. Elsevier, 2014. ISBN 978-0-08-100199-8. https:// doi.org/10.1533/9780081002452.1.3

Wood D (2015) The effect of rear geometry changes on the notchback flow field, $\mathrm{PhD}$ thesis, Loughborough University

Publisher's Note Springer Nature remains neutral with regard to jurisdictional claims in published maps and institutional affiliations. 